\title{
TOPOLOGICAL EXAMPLES OF PROJECTIVE MODULES
}

\author{
BY
}

\author{
RICHARD G. SWAN
}

\begin{abstract}
A new and more elementary proof is given for Lonsted's theorem that vector bundles over a finite complex can be represented by projective modules over a noetherian ring. The rings obtained are considerably smaller than those of Lønsted. In certain cases, methods associated with Hilbert's 17 th problem can be used to give a purely algebraic description of the rings. In particular, one obtains a purely algebraic characterization of the homotopy groups of the classical Lie groups. Several examples are given of rings such that all projective modules of low rank are free. If $m \equiv 2 \bmod 4$, there is a noetherian ring of dimension $m$ with nontrivial projective modules of rank $m$ such that all projective modules of rank $\neq m$ are free.
\end{abstract}

In [29], Lønsted proved a remarkable theorem which shows that vector bundles over a finite $\mathrm{CW}$ complex can be represented by finitely generated projective modules over a noetherian ring. This means that by purely topological constructions one can produce examples of noetherian rings whose projective modules have certain specified properties. This method has the advantage that one can impose conditions on the totality of projective modules while the more elementary method of [42] only allows us to construct a finite number of such modules at a time.

Lønsted's construction makes use of rather deep properties of analytic functions. In attempting to analyze his proof, I discovered a more elementary proof of the theorem which I will present here. This proof gives rings which are considerably smaller than the ones used by Lønsted. They are, in fact, localizations of algebras of finite type over $\mathbf{R}$. In certain cases, one can even give a very simple and purely algebraic description of the ring. This will be done in $\$ 10$ using methods associated with Hilbert's 17 th problem.

The starting point for this work was a question of A. Geramita. He pointed out that the rings in [42] have nontrivial projective modules of low rank and asked whether, for all $n$, there are noetherian rings having nontrivial projective modules but such that all such modules of rank less than $n$ are free. Three

Received by the editors December 9, 1975.

AMS (MOS) subject classifications (1970). Primary 13C10, 55F25, 12D15; Secondary 16A50, 55F50.

Key words and phrases. Projective modules, vector bundles. 
examples of this sort will be given in \$9. In contrast to [42], these examples are intrinsically topological and it is not at all clear how to construct the examples in a simple algebraic way. In [42] the examples were algebraic and topological methods were only used to prove nontriviality.

The basic idea for the new proof of Lønsted's theorem is to consider dense subrings of topological rings. This idea goes back to the elementary proof of periodicity by Atiyah and Bott [2] and particularly to the very algebraic exposition of this theorem given by Bass [5]. A number of ideas from this paper are used here. The essential content of Bass' paper was reproduced in [43, Chapter 17] but unfortunately without giving proper credit to Bass. Therefore, I would like to take this opportunity to correct this omission. The idea of looking at dense subrings was applied to $K$-theory by Evans [13]. A number of results of the present paper constitute nonstable generalizations of his work. A recent paper of B. Dayton gives further results in this direction, including a discussion of $K_{1}$ [9].

1. Topological rings. Let $R$ be a topological ring with unit. If $F$ and $G$ are free $R$-modules on $m$ and $n$ generators, we can identify $\operatorname{Hom}_{R}(F, G)$ with the set $M_{m n}(R)$ of $m \times n$ matrices over $R$ and therefore with $R^{m n}$. The product topology on $R^{m n}$ then induces a topology on $\operatorname{Hom}_{R}(F, G)$ which is easily seen to be independent of the choice of bases. If $P$ and $Q$ are finitely generated projective $R$-modules, we can find $P \oplus P^{\prime}=F, Q \oplus Q^{\prime}=G$ with $F$ and $G$ finitely generated free modules. Identify $\operatorname{Hom}_{R}(P, Q)$ with a submodule of $\operatorname{Hom}_{R}(F, G)$ by sending $f: P \rightarrow Q$ to $f \oplus 0: P \oplus P^{\prime} \rightarrow Q \oplus Q^{\prime}$. Give $\operatorname{Hom}_{R}(P, Q)$ the relative topology as a subset of $\operatorname{Hom}_{R}(F, G)$. Thus $g: P$ $\rightarrow Q$ is near $f: P \rightarrow Q$ if the matrix representing $(g-f) \oplus 0: F \rightarrow G$ has all entries near 0 . This topology is independent of the choices of $P^{\prime}$ and $Q^{\prime}$. To see this, we first observe that replacing $P^{\prime}$ and $Q^{\prime}$ by $P^{\prime} \oplus R^{r}, Q^{\prime} \oplus R^{s}$ trivially leads to the same topology. If $P \oplus P^{\prime \prime}=F^{\prime}$ and $Q \oplus Q^{\prime \prime}=G^{\prime}$, apply this remark to $f \oplus 0 \oplus 0 \oplus 0: P \oplus P^{\prime} \oplus P^{\prime \prime} \oplus P \rightarrow Q \oplus Q^{\prime} \oplus Q^{\prime \prime} \oplus Q$.

It is trivial to check that addition and composition are continuous in this topology. Note that we have also defined a topology on each finitely generated projective $R$-module because $P=\operatorname{Hom}_{R}(R, P)$. It is simply the relative topology on $P$ in $P \oplus P^{\prime}=F \approx R^{m}$.

We now make the following assumption:

(1) The group of units $R^{*}$ is open in $R$ and $u \mapsto u^{-1}$ is continuous on it.

This is equivalent to saying that all elements of $R$ sufficiently near 1 are units and have inverses near 1 . It is certainly true for Banach algebras [30, Theorem $22 \mathrm{~A}$ ] and is trivially true for rings of continuous real, complex, or quaternionic functions on a compact space.

Let $M_{n}(R)$ be the ring of $n \times n$ matrices over $R$ with the topology defined as above (note $M_{n}(R)=\operatorname{Hom}_{R}\left(R^{n}, R^{n}\right)$ ). Thus $\left(b_{i j}\right)$ is near $\left(a_{i j}\right)$ if $b_{i j}$ is near 
$a_{i j}$ for each $i j$. Clearly $M_{n}(R)$ is a topological ring.

Lemma 1.1. Suppose $R^{*}$ is open in $R$. Let $U$ be a neighborhood of 1 in $M_{n}(R)$. Then there is a neighborhood $V \subset U$ of 1 in $M_{n}(R)$ such that any matrix in $V$ can be reduced to diagonal form by at most $n(n-1)$ elementary row and column operations without ever leaving $U$.

PRoof. Let $M=\left(a_{i j}\right) \in M_{n}(R)$. If $M$ is very close to 1 , then $a_{11}$ is very near 1 and so is a unit of $R$. By elementary row and column operations we reduce $M$ to

$$
M^{\prime}=\left(\begin{array}{cccc}
a_{11} & 0 & \cdots & 0 \\
0 & & & \\
\vdots & & N & \\
0 & & &
\end{array}\right)
$$

This will still be very near 1 since $a_{11}^{-1}$ is near 1 . By induction on $n$, we can now reduce $M$ to diagonal form.

Corollary 1.2. If $R$ satisfies (1), so does $M_{n}(R)$.

Proof. The proof of Lemma 1.1 shows that if $x \in M_{n}(R)$ is near 1 we can write

$$
x=e_{1}(x) \cdots e_{r}(x) d(x) f_{1}(x) \cdots f_{r}(x)
$$

where $r=\frac{1}{2} n(n-1), d$ is diagonal, the $e_{i}$ and $f_{j}$ are elementary. The $e_{i}, f_{j}$ and $d$ are continuous in $x$ and reduce to 1 for $x=1$. Now

$$
x^{-1}=f_{r}(x)^{-1} \cdots f_{1}(x)^{-1} d(x)^{-1} e_{r}(x)^{-1} \cdots e_{1}(x)^{-1} .
$$

By (1) for $R, d^{-1}$ is defined and continuous in $d$ for diagonal matrices $d$ near 1 . There is no problem with the elementary matrices.

The next two lemmas show that something like invariance under homotopy holds if $R$ satisfies (1).

Lemma 1.3. Let $R$ satisfy (1). Let $P$ and $Q$ be finitely generated projective $R$ modules and let $f: P \rightarrow Q$ be surjective. If $g: P \rightarrow Q$ is sufficiently near $f$ then $g$ is surjective and $\operatorname{ker} g \approx \operatorname{ker} f$.

Proof. Let $s: Q \rightarrow P$ with $f s=1$. Let $Q \oplus Q^{\prime}=F \approx R^{n}$. If $g$ is near $f$ then $g s \oplus 1$ is near $f s \oplus 1=1_{F}$. By Corollary 1.2, gs $\oplus 1$ is invertible and so $g s$ is an isomorphism. Therefore $g$ is onto. Let $t=s(g s)^{-1}: Q \rightarrow P$. Then $g t=1_{Q}$ so $\operatorname{ker} g \approx \operatorname{ckr} t=\operatorname{ckr} s \approx \operatorname{ker} f$.

LEMMA 1.4. Assume $R$ satisfies (1). Let $F$ be a finitely generated projective $R$ - 
module and let $e: F \rightarrow F$ be idempotent. If $f: F \rightarrow F$ is idempotent and sufficiently near e, then $e(F) \approx f(F)$.

Proof. Let $P=\operatorname{im} e, P^{\prime}=\operatorname{im}(1-e), Q=\operatorname{im} f, Q^{\prime}=\operatorname{im}(1-f)$. If $F$ is very near $e$ the map $e+(1-f): F \rightarrow F$ will be very near $e+1-e=1_{F}$ and so, by Lemma 1.3, will be an isomorphism. Therefore $F=P+Q^{\prime}$ and we have an epimorphism $\alpha: P \rightarrow F / Q^{\prime}=Q$ given by $\alpha(x)=f(x)$ for $x \in P$. Let $\beta: Q \rightarrow P$ be defined by $\beta(y)=e(y)$. If $f$ is very near $e$, then $\beta \alpha: P \rightarrow P$ will be very near to the map $x \mapsto e^{2}(x)=x$. By Lemma $1.3, \beta \alpha$ will be an isomorphism and so $\alpha$ will also be injective.

Remark. Suppose $R$ is commutative and $R^{*}$ is open in $R$. If $x \in M_{n}(R)$ is near 1 , then det $x$ is near 1 so that det $x \in R^{*}$ and $x$ is invertible. Therefore $M_{n}(R)^{*}$ is open in $M_{n}(R)$. This shows that if $R$ is commutative we can dispense with the assumption that $u \mapsto u^{-1}$ is continuous in Lemmas 1.3 and 1.4. The same applies to the results of $\$ 2$ except for those dealing with $K_{1}$.

REMARK. Lemma 1.4 clearly fails if $f$ is not assumed idempotent, e.g. consider $e=0: R \rightarrow R$.

2. A comparison theorem. Consider now two rings $A$ and $B$ with unit and a ring homomorphism $\varphi: A \rightarrow B$ with $\varphi(1)=1$. Assume the following conditions hold.

(1) $B$ is a topological ring, $B^{*}$ is open in $B$ and $u \mapsto u^{-1}$ is continuous on $B^{*}$.

(2) $\varphi(A)$ is dense in $B$.

(3) If $\varphi(a)$ is sufficiently near 1 in $B$ then $a \in A^{*}$.

In the applications, we will usually have $A \subset B$ with $\varphi$ the inclusion map. However, the present formulation also includes the case where $B$ is discrete and of the form $B=A / I$ where $I$ is a radical ideal.

Most of the arguments are modeled on classical ones for this case. A similar situation is considered by Evans [13] and the results obtained here will generalize his Theorem 1. Evans uses the following construction: If $\varphi: A \rightarrow B$ satisfies (1) and (2), let $S=\varphi^{-1}\left(B^{*}\right)$. Then $A_{S} \rightarrow B$ satisfies (1), (2) and (3).

As in $\S 1$, we begin by checking that our hypotheses are inherited by matrix rings.

Lemma 2.1. If $\varphi: A \rightarrow B$ satisfies (1), (2) and (3), so does $M_{n}(\varphi): M_{n}(A)$ $\rightarrow M_{n}(B)$.

Proof. By Corollary 1.2, (1) is satisfied, while (2) is trivial. For (3), define a topology on $A$ by taking the neighborhoods of 0 to be the sets $\varphi^{-1}(U)$ where $U$ is a neighborhood of 0 in $B$. Then (3) holds if and only if $A$ satisfies (1) of $\S 1$, provided that $B$ satisfies (1). The continuity of $u \mapsto u^{-1}$ is clear because $\varphi\left(u^{-1}\right)=\varphi(u)^{-1}$ is near 1 whenever $\varphi(u)$ is near 1 . By applying Corollary 1.3 to $A$ we see that $M_{n}(\varphi)$ satisfies (3). 
If $R$ is any ring with unit, let $P(R)$ denote the monoid of isomorphism classes of finitely generated projective $R$-modules. It is a functor. If $\varphi: A \rightarrow B$ then $P(\varphi): P(A) \rightarrow P(B)$ by $(P) \mapsto\left(B \otimes_{A} P\right)$.

The symbols "sr" and "psr" denote the stable range and projective stable range [44], [45]. See also $\$ 8$.

Let $u(R)$ denote the image of $R^{*}=G L_{1}(R) \rightarrow K_{1}(R)$. If $R$ is commutative, $u(R)=R^{*}$ and $K_{1}(R)=u(R) \oplus S K_{1}(R)$.

Theorem 2.2. Let $\varphi: A \rightarrow B$ satisfy (1), (2) and (3). Then

(a) $P(\varphi): P(A) \rightarrow P(B)$ is injective and $(P)$ lies in the image if and only if $[P] \in \operatorname{im}\left[K_{0}(A) \rightarrow K_{0}(B)\right]$.

(b) $K_{1}(B)=\operatorname{im} K_{1}(\varphi)+u(B)$ and $\operatorname{ker} K_{1}(\varphi) \subset u(A)$. Therefore $S K_{1}(\varphi)$ is an isomorphism if $A$ and $B$ are commutative.

(c) $\operatorname{sr} A \leqslant \operatorname{sr} B$ and $\operatorname{psr} A \leqslant \operatorname{psr} B$.

Note that (a) implies that $K_{0}(A) \rightarrow K_{0}(B)$ is injective. This is Evans' result [13]. The characterization of the image was suggested by Eisenbud's remark that the tangent bundle of $S^{n}$ can be lifted to a projective module over $\mathbf{R}\left[x_{0}, \ldots, x_{n},\left(\sum x_{i}^{2}\right)^{-1}\right]$. This suggested the following construction. If $I$ is an ideal of a commutative ring $R$, let $S=1+I$. Then $I_{S}$ is a radical ideal of $R_{S}$ and $R_{S} / I_{S}=R / I$. By Theorem 2.2(a), a finitely generated projective $R / I$ module can be lifted to one over $R_{S}$ if and only if this can be done stably. In particular, all stably free modules can be lifted to $R_{S}$.

We now turn to the proof of Theorem 2.2 and begin with (b). If $x \in G L_{n}(A)$ and $\varphi(x) \in E_{n}(B)$, write $\varphi(x)=\prod e_{i_{,} j_{\nu}}\left(b_{\nu}\right)$. Choose $a_{\nu} \in A$ with $\varphi\left(a_{\nu}\right)$ very near $b_{\nu}$ and let $y=x\left[\Pi e_{i_{\nu} j_{\nu}}\left(a_{\nu}\right)\right]^{-1}$. Then $\varphi(y)$ is very near 1 . As in the proof of Lemma 2.1, we can use the procedure of Lemma 1.1 to reduce $y$ to diagonal form. By [6, Chapter V, Corollary 1.8a], $y$ $\in E(A) G L_{1}(A)$.

If $x \in G L_{n}(B)$, find $y \in M_{n}(A)$ with $\varphi(y)$ very near $x$ and $z \in M_{n}(A)$ with $\varphi(z)$ very near $x^{-1}$. Then $\varphi(y z)$ and $\varphi(z y)$ are very near 1 . By Lemma 2.1, $y z$ and $z y \in G L_{n}(A)$ and so $y \in G L_{n}(A)$. Now $x^{-1} \varphi(y)$ is near 1 so $x^{-1} \varphi(y)$ $\in E_{n}(B) G L_{1}(B)$ as above.

Lemma 2.3. Let $P$ and $Q$ be finitely generated projective $A$-modules and let $f: B \otimes_{A} P \rightarrow B \otimes_{A} Q$. Then we can find $g: P \rightarrow Q$ such that $B \otimes_{A} g$ is arbitrarily close to $f$.

Proof. Let $P \oplus P^{\prime}=F$ and $Q \oplus Q^{\prime}=G$ be free and let $f \oplus 0: B \otimes F$ $\rightarrow B \otimes G$ be represented by a matrix $\left(b_{i j}\right)$. Find $a_{i j} \in A$ with $\varphi\left(a_{i j}\right)$ near $b_{i j}$ and let $h: F \rightarrow G$ be defined by $\left(a_{i j}\right)$. Let $g$ be the composition $P \rightarrow^{i} F \rightarrow^{h} G$ $\rightarrow{ }^{p} Q$ where $i$ is the inclusion and $p$ is the projection.

Using this, we can now prove (c). Let $P$ be a finitely generated projective $A$ - 
module with $\mathrm{rk} P \geqslant \operatorname{psr} B$. Let $(a, \pi) \in A \oplus P$ be unimodular. Find $\eta \in B$ $\otimes P$ so that $1 \otimes \pi+\varphi(a) \eta$ is unimodular in $B \otimes P$. Let $f: B \otimes P \rightarrow B$ with $f(1 \otimes \pi+\varphi(a) \eta)=1$. Apply Lemma 2.3 to $B \otimes A=B \rightarrow^{\eta} B \otimes P$ and $B \otimes P \rightarrow f \quad B$ to get $\xi \in P, g: P \rightarrow A$ with $1 \otimes \xi$ near $\eta$ and $B \otimes g$ near $f$. If $g(\pi+a \xi)=x$, then $\varphi(x)$ is near $f(1 \otimes \pi+\varphi(a) \eta)=1$. If the approximation is good enough, $x$ will be a unit of $A$ by (3) and so $\pi+a \xi$ will be unimodular. Therefore, $\operatorname{psr} A \leqslant \operatorname{psr} B$. The same argument with $P$ free gives $\operatorname{sr} A \leqslant \operatorname{sr} B$.

REMARK. In the main case of interest, these inequalities are actually equalities (see §8) but I do not see how to prove this in general.

To prove (a), we use the following extension of Nakayama's lemma.

Lemma 2.4. Let $\varphi: A \rightarrow B$ satisfy (1), (2) and (3). Let $M$ be a finitely generated $A$-module. If $B \otimes_{A} M=0$, then $M=0$.

Proof. Find a resolution $F^{\prime} \rightarrow{ }^{f} F \rightarrow M \rightarrow 0$ with $F=A^{n}, F^{\prime}$ free. Since $B \otimes M=0, B \otimes f$ is onto and we can find $s: B \otimes F \rightarrow B \otimes F^{\prime}$ with $(B \otimes f) s$ $=1$. Some finite subset of a base for $F^{\prime}$ generates a free submodule $G$ with $s: B \otimes F \rightarrow B \otimes G$. By Lemma 2.3, find $t: F \rightarrow G$ with $B \otimes t$ near $s$. Then $B \otimes f t$ is near $(B \otimes f) s=1$. By Lemma 2.1, $M_{n}(\varphi)$ satisfies (3). Therefore $f t$ will be an isomorphism if $B \otimes t$ is close enough to $s$ and hence $f$ is onto.

To prove (a), suppose $f: B \otimes P \approx B \otimes Q$. By Lemma 2.3, find $g: P \rightarrow Q$ with $B \otimes g$ near $f$. By Lemma $1.3, B \otimes g$ will be an isomorphism. If $M=\operatorname{ckr} g$, then $B \otimes M=0$ so $M=0$ by Lemma 2.4. If $N=\operatorname{ker} g$ we have a split exact sequence $0 \rightarrow N \rightarrow P \rightarrow Q \rightarrow 0$. Since $N$ is finitely generated as a summand of $P$, and $B \otimes N=0$ since $B \otimes g$ is an isomorphism, we see that $N=0$ by Lemma 2.4. This argument is, of course, a variant of the classical projective cover argument.

Finally, let $[P] \in \operatorname{im}\left[K_{0}(A) \rightarrow K_{0}(B)\right]$ be the image of $\xi \in K_{0}(A)$. Write $-\xi=[Q]-[F]$. Then $B \otimes F$ is stably isomorphic to $P \oplus(B \otimes Q)$. By adding a free module to $F$ and $Q$ we can suppose that $B \otimes F \approx P \oplus(B \otimes Q)$. Let $p: B \otimes F \rightarrow B \otimes Q$ be the projection. By Lemma 2.3 find $g: F \rightarrow Q$ with $B \otimes g$ very near $p$. Then Lemma 1.3 shows that $B \otimes g$ is onto. As above, $g$ is then onto by Lemma 2.4. Therefore $S=\operatorname{ker} g$ is a summand of $F$ and hence projective. Now $B \otimes S=\operatorname{ker} B \otimes g \approx \operatorname{ker} p=P$ by Lemma 1.3 if $B \otimes g$ is close enough to $p$.

3. Surjectivity. Examples given below show that $P(\varphi): P(A) \rightarrow P(B)$ need not be onto in Theorem 2.2. If $B=A / I$ where $A$ is $I$-adically complete, the surjectivity of $P(\varphi)$ is shown by using an idempotent lifting argument [43, Theorem 2.26], [6, Chapter III, Proposition 2.12]. I will give a sufficient condition for surjectivity using one of the classical methods for lifting idempotents [26, Chapter III, §8].

Suppose $\varphi: A \rightarrow B$ satisfies (1), (2) and (3) of $\S 2$. As in $\$ 2$, define a topology 
on $A$ using that induced from $B$ so that a base of neighborhoods for 0 consists of all $\varphi^{-1}(U)$ where $U$ is a neighborhood of 0 in $B$. For each integer $n$ consider the following condition

$\left(\mathrm{SBI}_{n}\right)$ : If $U$ is a neighborhood of 0 in $A$, there exists a neighborhood $V$ of 0 in $A$ with the following property:

If $z \in M_{n}(A)$ has all entries in $V$, there is some $x \in M_{n}(A)$ with all entries in $U$ such that

(a) $x^{2}-x=z$.

(b) Every $y \in M_{n}(A)$ which commutes with $z$ also commutes with $x$.

THEOREM 3.1. Suppose $\varphi: A \rightarrow B$ satisfies (1), (2), (3) and $\left(\mathrm{SBI}_{n}\right)$ for all $n$. Then $P(\varphi): P(A) \rightarrow P(B)$ is bijective.

Proof. Let $P$ be a finitely generated projective $B$-module. Let $P \oplus P^{\prime}=F$ $\approx B^{n}$ and let $e: F \rightarrow F$ be the projection on $P$. Then $e \in M_{n}(B)$ and $e^{2}=e$. Choose $u \in M_{n}(A)$ with $\varphi(u)$ very close to $e$. If $z=u^{2}-u$ then $\varphi(z)$ is very close to 0 . Therefore $\varphi(1+4 z)$ is near 1 so $1+4 z$ is invertible by Lemma 2.1 and $\varphi\left((1+4 z)^{-1}\right)=\varphi(1+4 z)^{-1}$ is near 1 by Corollary 1.2. Let $t=-z(1$ $+4 z)^{-1}$. Then $\varphi(t)$ is very close to 0 . By $\left(\mathrm{SBI}_{n}\right)$, solve $x^{2}-x=t$ with $x$ near 0 . Let $f=u+(1-2 u) x \in M_{n}(A)$. As in [26, Chapter III, \&8] we see easily that $f^{2}=f$. We can make $\varphi(f)$ arbitrarily close to $e$ by taking $U$ in $\left(\mathrm{SBI}_{n}\right)$ small enough and taking $\varphi(u)$ sufficiently near $e$ so that $t \in V$ of $\left(\mathrm{SBI}_{n}\right)$. By Lemma 1.4, if $\varphi(f)$ is close enough to $e$, then $\operatorname{im} \varphi(f) \approx \operatorname{im} e=P$. But if $Q=\operatorname{im} f$, then $\operatorname{im} \varphi(f) \approx B \otimes_{A} Q$.

If $B=A / I$ where $A$ is $I$-adically complete, we can prove $\left(\mathrm{SBI}_{n}\right)$ by the argument in [26, Chapter III, \$8] and recover the standard result that $P(A) \cong P(B)$ in this case.

From now on it will be convenient to assume commutativity. In order to include the quaternionic case, I will proceed as follows. Let $\varphi: A \rightarrow B$ satisfy (1), (2) and (3) of $\S 2$. Assume $A$ and $B$ commutative. Let $k \subset A$ be a subring and let $\Lambda$ be a $k$-algebra which is finitely generated and free as a $k$-module. We then consider the map $\psi=\varphi \otimes 1: A \otimes_{k} \Lambda \rightarrow B \otimes_{k} \Lambda$. In the applications, $k=\mathbf{R}$ and $\Lambda=\mathbf{R}, \mathbf{C}$, or $\mathbf{H}$.

Choosing a base for $\Lambda$ over $k$ gives us a module isomorphism $B \otimes_{k} \Lambda \approx B^{n}$. We topologize $B \otimes \Lambda$ by using the product topology on $B^{n}$. This is clearly independent of the choice of base and makes $B \otimes_{k} \Lambda$ a topological ring.

Lemma 3.2. $\psi: A \otimes \Lambda \rightarrow B \otimes \Lambda$ satisfies (1), (2) and (3) of $\S 2$.

Proof. It is clear that (2) holds. For (1), let $b \in B \otimes \Lambda$ be close to 1 . Let $f: B \otimes \Lambda \rightarrow B \otimes \Lambda$ by $f(x)=x b$. Identify $B \otimes \Lambda$ with $B^{n}$ as above. Then $f \in M_{n}(B)$. If $b$ is near 1 then $f$ is near 1 so $f^{-1}$ exists and is near 1 in $M_{n}(B)$ by Corollary 1.2. Let $f^{-1}(1)=c$. Then $c$ is near 1 because $f$ is near 1 . Now 
$1=f\left(f^{-1}(1)\right)=f(c)=c b$. The same argument on $g(x)=b x$ shows that $b$ has a right inverse. Therefore $b^{-1}$ exists and $f^{-1}(x)=x b^{-1}$ so that $b^{-1}=c$ is close to 1 .

For (3) we can argue as in Lemma 1.1. Alternatively, if $a \in A \otimes \Lambda$ is near 1 and $f(x)=x a$, then $\operatorname{det}(f)$ is near 1 so $f^{-1}$ exists. As above we get $c a=1$ with $c=f^{-1}(1)$ and, similarly, $a$ has a right inverse.

REMARK. If $\varphi$ satisfies the stronger condition

$$
\varphi^{-1}\left(B^{*}\right)=A^{*}
$$

which clearly implies (3) in the presence of (1), then $\psi$ also satisfies $\left(3^{\prime}\right)$. This follows from the last argument above.

In the present situation, we can dispence with (b) of $\left(\mathrm{SBI}_{n}\right)$.

Lemma 3.3. Let $\varphi: A \rightarrow B$ satisfy (1), (2) and (3). Let $k$ and $\Lambda$ be as above. Then, for given $n$, there is a neighborhood $U$ of 0 in $A$ with the following property: If $x \in M_{n}(A)$ has all entries in $U$, then each $y \in M_{n}(A)$ which commutes with $x^{2}-x$ also commutes with $x$.

Proof. Let $z=[x, y]=x y-y x$. If $\left[x^{2}-x, y\right]=0$ then $z=$ $[x, y]=\left[x^{2}, y\right]=x z+z x$. Let $z_{1}, \ldots, z_{n^{2}}$ be the entries of $z$ in some order. Since $A$ is commutative, the equation $z=x z+z x$ can be written in the form $z_{i}=\sum a_{i j} z_{j}$ where each $a_{i j}$ is a sum of entries of $x$. Let $\zeta=\left(z_{i}\right)$ and $\Delta=\left(\delta_{i j}-\right.$ $\left.a_{i j}\right)$. Then our equation reads $\Delta \zeta=0$. But, if $\varphi(x)$ is close to 0 , so are all $\varphi\left(a_{i j}\right)$. Therefore $\varphi(\Delta)$ is near 1 and $\Delta$ is invertible by Lemma 2.1. It follows that $\zeta=0$ and so $z=0$. The smallness condition on $\varphi(x)$ is clearly independent of $y$.

Lemma 3.4. If $\varphi: A \rightarrow B$ satisfies (1), (2), (3) and $\left(\mathrm{SBI}_{n}\right)(\mathrm{a})$ for all $n$, then $\psi=\varphi \otimes 1: A \otimes \Lambda \rightarrow B \otimes \Lambda$ satisfies $\left(\mathrm{SBI}_{n}\right)$ for all $n$.

Proof. Suppose $z \in M_{n}(A \otimes \Lambda)$. By choosing a base $e_{1}, \ldots, e_{r}$ for $\Lambda$ we get an embedding

$$
\begin{aligned}
M_{n}(A \otimes \Lambda) & =\operatorname{Hom}_{A \otimes \Lambda}(A \otimes \Lambda, A \otimes \Lambda) \subset \operatorname{Hom}_{A}(A \otimes \Lambda, A \otimes \Lambda) \\
& =M_{r n}(A) .
\end{aligned}
$$

If $E_{i} \in M_{r n}(A)$ is multiplication by $1 \otimes e_{i}$, then $M_{n}(A \otimes \Lambda)$ is the centralizer in $M_{r n}(A)$ of $E_{1}, \ldots, E_{r}$. Now if $z \in M_{n}(A \otimes \Lambda)$ is near 0, we can solve $x^{2}-x=z$ in $M_{r n}(A)$. If we keep close enough to 0, Lemma 3.3 applies. Since $\left[E_{i}, z\right]=0$ we get $\left[E_{i}, x\right]=0$ so $x \in M_{n}(A \otimes \Lambda)$. Clearly $\left(\mathrm{SBI}_{n}\right)(\mathrm{b})$ also holds.

THEOREM 3.5. Let $\varphi: A \rightarrow B$ be a map of commutative rings satisfying (1), (2), (3) and $\left(\mathrm{SBI}_{n}\right)(\mathrm{a})$. Let $\Lambda$ be a k-algebra, $k \subset A$, which is finitely generated and free as a $k$-module. Then $P(\varphi \otimes 1): P(A \otimes \Lambda) \rightarrow P(B \otimes \Lambda)$ is bijective. 
4. Approximating SBI. Suppose now that $\varphi$ is an inclusion $A \subset B$ of commutative rings satisfying (1), (2) and (3'), i.e. $A^{*}=A \cap B^{*}$. Let $k$ and $\Lambda$ be as in Theorem 3.5. I will assume now only that $B$ itself satisfies $\left(\mathrm{SBI}_{n}\right)(\mathrm{a})$, i.e.

(4) If $n$ is a given integer and $U$ is a neighborhood of 0 in $B$, there is a neighborhood $V$ of 0 in $B$ with the following property:

If $z \in M_{n}(B)$ has all entries in $V$, there is an $x \in M_{n}(B)$ with all entries in $U$ such that $x^{2}-x=z$.

Suppose we are given $P \in P(B \otimes \Lambda)$. We want to enlarge $A$ to a ring $A_{1} \subset B$ still satisfying (1), (2) and (3'), and such that $P$ lies in the image of $P(A \otimes \Lambda) \rightarrow P(B \otimes \Lambda)$. To do this following the proof of Theorem 3.1 we have to solve an equation $x^{2}-x=t$ in $M_{n}(B \otimes \Lambda)$. As in the proof of Lemma 3.4, solve the equation in $M_{r n}(B)$ using (4). If we keep $t$, and hence $x$, very close to 0 , it follows that $x \in M_{n}(B \otimes \Lambda)$ as in Lemma 3.4. Adjoin all entries of $x \in M_{r n}(B)$ to $A$ getting $A^{\prime} \subset B$, and let $A_{1}=A_{S}^{\prime}$, where $S=A^{\prime}$ $\cap B^{*}$. We can then conclude that $P$ is in the image of

$$
P\left(A_{1} \otimes \Lambda\right) \rightarrow P(B \otimes \Lambda)
$$

as in the proof of Theorem 3.1. The closeness of approximation needed to make the proof work depended only on $B$ and $P$, and not on the choice of $A$.

Note that $A_{1}$ is essentially of finite type over $A$, i.e., a localization of an $A$ algebra of finite type. Therefore $A_{1}$ is noetherian if $A$ is.

We would like to be able to perform this construction without increasing the dimension of $A$. If $x_{1}, \ldots, x_{N}, N=(r n)^{2}$ are the entries of $x \in M_{r n}(B)$ in some order, we can write the equation $x^{2}-x=t$ in the form $f_{i}(x)=x_{i}$ $+\sum a_{i j} x_{i} x_{j}=-t_{i}$ where $a_{i j} \in \mathbf{Z}$. The Jacobian

$$
J=\left|\partial\left(f_{1}, \ldots, f_{N}\right) / \partial\left(x_{1}, \ldots, x_{N}\right)\right|
$$

has the form $\operatorname{det}\left(1+\sum c_{i} x_{i}\right)$ where $c_{i} \in M_{N}(\mathbf{Z})$. In our construction we can take $t$ and hence $x$ as close to 0 as we like. Therefore, we can make $J$ so close to 1 that it is a unit in $B$. Then $A_{1}$ is a localization of $A^{\prime}\left[J^{\prime}\right]$. Therefore, it follows by standard results on étale extensions [20, Exposé 1] that $\operatorname{dim} A_{1}$ $\leqslant \operatorname{dim} A$. Alternatively, we can argue as follows. If $P$ is a prime ideal of $A_{1}$ and $F \subset K$ are the quotient fields of $A / A \cap P$ and $A_{1} / P$, then

$$
K=F\left(\xi_{1}, \ldots, \xi_{N}\right)
$$

where $\xi_{i}$ is the image of $x_{i}$ in $K$. Now $f_{i}(\xi)=-\tau_{i} \in F$. If $D$ is a derivation of $K$ over $F$, then

$$
D f_{i}(\xi)=\sum \frac{\partial f_{i}}{\partial x_{j}}(\xi) D \xi_{j}=0 .
$$

The matrix of coefficients has determinant $J$ which is a unit in $A_{1}$ and hence 
in $K$. Therefore $D \xi_{i}=0$ for all $i$ so $D=0$. This means that $K$ is separable algebraic over $F$ [48, Chapter II, §17, Theorem 40].

If $R \subset R_{1}$ are commutative rings, define

$$
t\left(R_{1} / R\right)=\sup \operatorname{transc}\left(\mathrm{qf}\left(R_{1} / P\right) / \mathrm{qf}(R / R \cap P)\right)
$$

where $P$ runs over the prime ideals of $R_{1}$ and "qf" denotes the quotient field.

We have just shown that $t\left(A_{1} / A\right)=0$ so $\operatorname{dim} A_{1} \leqslant \operatorname{dim} A$ by Lemma 5.3 below.

By iterating the above construction, we arrive at the following result.

THEOREM 4.1. Let $B$ be a commutative ring satisfying (1) and (4). Let $R \subset B$ be a dense subring. Let $\Lambda_{1}, \ldots, \Lambda_{n}$ be $k$-algebras $k \subset R$, which are finitely generated and free as $k$-modules. Let $G_{i} \subset K_{0}\left(B \otimes \Lambda_{i}\right), i=1, \ldots, n$, be finitely generated subgroups. Then we can find a subring $A \subset B$ with $R \subset A$ such that

(a) $A \subset B$ satisfies (1), (2) and (3'),

(b) $\operatorname{im}\left[P\left(A \otimes \Lambda_{i}\right) \rightarrow P\left(B \otimes \Lambda_{i}\right)\right]$ contains all $P_{i}$ with $\left[P_{i}\right] \in G_{i}$,

(c) $A$ is essentially of finite type over $R$,

(d) $t(A / R)=0$ and hence $\operatorname{dim} A \leqslant \operatorname{dim} R$.

Proof. First replace $R$ by $R_{S}$ with $S=R \cap B^{*}$. Then apply the above construction to each of a finite collection $P_{j}$ of projectives which generate all $G_{i}$. Then apply Theorem 2.2. For (d) use Lemmas 5.1 and 5.3.

5. Transcendency and dimension. I will prove here a few elementary facts used in $\S 4$ and $\S 6$. Let $t\left(R_{1} / R\right)$ be as in $\S 4$.

LEMMA 5.1. If $A \subset B \subset C$, then $t(C / A) \leqslant t(B / A)+t(C / B)$.

This is immediate from the definition. The example below shows that equality need not hold.

LEMMA 5.2. If $k$ is a field and $k \subset A \subset B$ then $t(A / k) \leqslant t(B / k)$.

Proof. Let $P$ be a prime ideal of $A$. Choose $Q \subset B$ maximal such that $Q \cap(A-P)=\varnothing$. Then $Q$ is prime and $Q \cap A \subset P$. Now

$$
\operatorname{transc}(\operatorname{qf}(A / A \cap Q) / k) \leqslant \operatorname{transc}(\operatorname{qf}(B / Q) / k) \leqslant t(B / k) .
$$

Since $A / P$ generates $\mathrm{qf}(A / P)$ as an extension of $k$, we can find $x_{1}, \ldots, x_{n}$ $\in A / P, n=\operatorname{transc}(\mathrm{qf}(A / P) / k)$ which are algebraically independent over $k$. Lift $x_{i}$ to $y_{i} \in A / A \cap Q$. The $y_{i}$ are algebraically independent over $k$ so

$$
n \leqslant \operatorname{transc}(\mathrm{qf}(A / A \cap Q) / k) \leqslant t(B / k) \text {. }
$$

The example below shows that this lemma is false if $k$ is not assumed to be a field. 
Lemma 5.3. If $t(B / A)=0$, then $\operatorname{dim} A \leqslant \operatorname{dim} B$.

Proof. Let $P_{0}<\cdots<P_{n}$ be a chain of prime ideals in $B$. I claim that the $P_{i} \cap A$ are distinct. Suppose $P<Q$ in $B$ and $P \cap A=Q \cap A$. Since $t((B / P) /(A / A \cap P))=0$ we can consider $A / A \cap P \subset B / P$. Thus we can assume that $A$ and $B$ are domains and $Q \cap A=0$. If $Q \neq 0$, let $x \in Q, x$ $\neq 0$. Since $x$ is algebraic over the quotient field of $A$, it satisfies a nontrivial equation $a_{0} x^{n}+\cdots+a_{n}=0, a_{i} \in A, a_{0} \neq 0$. Choose $n$ least. Then $a_{n} \neq 0$ since $B$ is a domain and $x \neq 0$. Therefore $a_{n} \in A \cap Q$ so $A \cap Q \neq 0$. This argument is essentially the same as the classical one used when $B$ is integral over $A$.

Note that equality need not hold, e.g. if $B$ is the quotient field of $A$.

EXAMPLE. Let $k$ be a field,

and

$$
A=k[x, y], \quad B=k[x, y, y / x]=k[x, y, z] /(x z-y)
$$

$$
C=k(x, y) .
$$

This corresponds to blowing up a point of the plane. Taking $P=B x$, we have $B / P=k[z]$ but $A / P \cap A=k$ since $y \in P$. Therefore, $t(B / A) \geqslant 1$ but $t(C / B)=t(C / A)=0$.

The following partial generalization of Lemma 5.3 is included for completeness. It is presumably well known.

THEOREM 5.4. Let $A \subset B$ be commutative domains with quotient fields $F \subset K$. If $A$ is noetherian, then

$$
\operatorname{dim} B \leqslant \operatorname{dim} A+\operatorname{transc}(K / F) .
$$

Proof. Let $P_{0}<\cdots<P_{n}$ be a chain of prime ideals in $B$. Let $x_{i} \in P_{i}$ $-P_{i-1}$. If $C=A\left[x_{1}, \ldots, x_{n}\right]$, then the $P_{i} \cap C$ are distinct. Therefore it is enough to consider the case where $B$ is of finite type over $A$. By induction, we reduce immediately to the case where $B=A[\xi]$. If $\xi$ is transcendental over $A$ then $\operatorname{dim} A[\xi]=1+\operatorname{dim} A$ by [35, Chapter I, 9.10] or [27, Theorem 149]. If not, $B=A[x] / P$ where $P \neq 0$. By lifting a chain of primes in $B$ back to $A[x]$ and putting 0 at the bottom, we see that $\operatorname{dim} B \leqslant \operatorname{dim} A[x]-1=\operatorname{dim} A$.

COROLlaRy 5.5. If $A \subset B$ are commutative rings with $A$ noetherian, then $\operatorname{dim} B \leqslant \operatorname{dim} A+t(B / A)$.

In fact we can replace $t(B / A)$ here by the analogous expression in which the sup is taken only over minimal prime ideals of $B$.

Note that strict inequality can occur, for example if $A$ is local and $B=A\left[a^{-1}\right]$ with $a \in A$ a nonunit. 
The argument from the case $B=A[x]$ to the general case is purely formal. Examples due to Seidenberg [39] show that the theorem fails for $B=A[x]$ if $A$ is not assumed noetherian.

6. Lonsted's theorem. We now specialize to the case $B=\mathbf{R}^{X}$, the ring of continuous real valued functions on a compact space $X$. It is a Banach algebra under the sup norm. $\|f\|=\sup |f(x)|$ and trivially satisfies (1).

Lemma 6.1. If $X$ is compact, $B=\mathbf{R}^{X}$ satisfies (4) of $\$ 4$.

Proof. The map $M_{n}(\mathbf{R}) \rightarrow M_{n}(\mathbf{R})$ by $x \mapsto x^{2}-x$ has Jacobian \pm 1 at $x=0$ and hence has a local inverse $f$ by the implicit function theorem i.e. there is a neighborhood $W$ of 0 in $M_{n}(\mathbf{R})$ and $f: W \rightarrow M_{n}(\mathbf{R})$ such that $f(x)^{2}-f(x)=x$ and $f(0)=0$. If $z \in M_{n}(B)$, regard $z$ as a map $z: X$ $\rightarrow M_{n}(\mathbf{R})$. If $z$ is near 0 it maps $X$ into $W$ and we can define $x=f \circ z$.

Suppose now that $X$ is a finite polyhedron. The spectral sequence of [4], [11] shows that $K_{\mathrm{R}}^{0}(X), K_{\mathrm{C}}^{0}(X), K_{\mathrm{H}}^{0}(X)$ are finitely generated. By [42], these are the same as $K_{0}(B), K_{0}\left(\mathbf{C} \otimes_{\mathbf{R}} B\right), K_{0}\left(\mathbf{H} \otimes_{\mathbf{R}} B\right)$. We can now apply Theorem 4.1. If we embed $X$ in some Euclidean space $\mathbf{R}^{m}$, the ring $R$ of polynomial functions on $X$ will be dense in $B$ by the Stone-Weierstrass theorem [17], [30].

LEMMA 6.2. Let $X$ be a finite polyhedron rectilinearly embedded in $\mathbf{R}^{m}$. Let $R$ be the ring of polynomial functions on $X$. Then $\operatorname{dim} R=t(R / R)=\operatorname{dim} X$.

Proof. Let $\sigma$ be an $r$-simplex and let $R_{\sigma}$ be the ring of polynomial functions on $\sigma$. Clearly $R_{\sigma} \approx \mathbf{R}\left[x_{1}, \ldots, x_{r}\right]$. Let $P_{\sigma}$ be the kernel of the restriction $R \rightarrow R_{\sigma}$. Then $\cap P_{\sigma}=0$ since $R \rightarrow \prod R_{\sigma}$ is injective. If $P$ is a prime ideal of $R$, then $\Pi P_{\sigma}=0 \subset P$ so some $P_{\sigma} \subset P$. Therefore $R / P=R_{\sigma} /\left(P / P_{\sigma}\right)$. Since we can also take $P=P_{\sigma}$, the result is clear.

We now get the following extension of Lønsted's theorem.

THEOREM 6.3. Let $X$ be a finite simplicial complex. Then there is a subring $A \subset \mathbf{R}^{X}$ with $\mathbf{R} \subset A$ such that

(a) $A \subset \mathbf{R}^{X}, \mathbf{C} \otimes_{\mathrm{R}} A \subset \mathbf{C}^{X}$, and $\mathbf{H} \otimes_{\mathbf{R}} A \subset \mathbf{H}^{X}$ satisfy (1), (2) and (3'),

(b) $P(A) \cong P\left(\mathbf{R}^{X}\right), P\left(\mathbf{C} \otimes_{\mathbf{R}} A\right) \cong P\left(\mathbf{C}^{X}\right)$, and $P\left(\mathbf{H} \otimes_{\mathbf{R}} A\right) \cong P\left(\mathbf{H}^{X}\right)$,

(c) $A$ is essentially of finite type over $\mathbf{R}$ and therefore noetherian,

(d) $\operatorname{dim} A \leqslant t(A / \mathbf{R})=\operatorname{dim} X$.

REMARKS. (1) Lønsted embeds $X$ rectilinarly and in general position and chooses $A$ to be the ring of functions on $X$ which extend to real analytic functions on a neighborhood of $X$. He uses fairly deep results on analytic functions to show that $A$ is noetherian and $\operatorname{dim} A=\operatorname{dim} X$. It is very easy to see that Theorem 3.5 applies to $A$. Only the SBI contition is not obvious. But, in the proof of Lemma $6.1, f$ is analytic and so $x$ is analytic if $z$ is. The same applies to the ring of Nash functions considered by Lønsted in [28], 
(2) If $X$ is a compact set in $\mathbf{R}^{m}, R$ the ring of polynomial functions on $X$, and if $K_{0}(R) \rightarrow K^{0}(X)$ is known to be onto, we can simply take $A=R_{S}$, where $S$ is the set of polynomial functions which are never 0 on $X$. For example, if $X$ is the $n-1$ sphere in $\mathbf{R}^{n}$, this will work by a theorem of Fossum [15] (see §11). If instead we let $R=\mathbf{R}\left[x_{1}, \ldots, x_{m}\right]$ and let $S$ be the set of polynomials having no zeros on $X$, then $R_{S} \rightarrow \mathbf{R}^{X}$ satisfies (1), (2) and (3'). Since $K_{0}\left(R_{S}\right)=G_{0}\left(R_{S}\right)$ and $\mathbf{Z}=G_{0}(R) \rightarrow G_{0}\left(R_{S}\right)$ is onto [6] we have $K_{0}\left(R_{S}\right)=\mathbf{Z}$. Therefore $\operatorname{im}\left[P\left(R_{S}\right) \rightarrow P\left(\mathbf{R}^{X}\right)\right]$ consists exactly of the stably free modules. If Int $X \neq \varnothing$, then $\mathbf{R}\left[x_{1}, \ldots, x_{m}\right]$ will actually be the ring of polynomial functions on $X$, e.g. if $X$ is an $m$-ball or a spherical shell in $\mathbf{R}^{m}$.

(3) We can also recover a special case of a theorem due to Forster [14, Theorem 6]. He shows that if $A$ is a commutative Banach algebra with unit and $X$ is its maximal ideal space, then $P(A) \rightarrow \stackrel{\approx}{ } P\left(\mathbf{C}^{X}\right)$. This is a very deep result as the proof depends on Grauert's theorem on vector bundles over Stein manifolds. However, if $A$ is selfadjoint, it can be proved in an elementary way. If $B=\mathbf{C}^{X}$, the Gelfand map $\varphi: A \rightarrow B$ clearly satisfies (1) and (3). Since $A$ is selfadjoint, it satisfies (2) $\left[30, \S 26 \mathrm{~B}\right.$, Lemma 1]. To prove $\left(\mathrm{SBI}_{n}\right)(\mathrm{a})$, we can reduce to the case where $A$ is semisimple and apply the Arens-Calderón theorem as in [14].

(4) If $X$ is a compact, finite-dimensional metric space of dimension $d$ we can embed $X$ in $\mathrm{R}^{2 d+1}$ and proceed as above provided $K^{0}(X)$ is finitely generated. The result will be similar to Theorem 6.3 but with (d) replaced by $\operatorname{dim} A$ $\leqslant t(A / \mathbf{R}) \leqslant 1+2 \operatorname{dim} X$ and will, of course, require finite generation of $K^{0}$ as a hypothesis. On the other hand, if we allow $A$ to be nonnoetherian, Theorem 6.3 extends to all compact metric spaces.

THEOREM 6.4. Let $X$ be a compact metric space. Then there is a subring $A \subset \mathbf{R}^{X}$ with $\mathbf{R} \subset A$ satisfying (a), (b) and (d) of Theorem 6.3 and, in addition, $\left(c^{\prime}\right) A$ is essentially of countable type over $R$.

In other words, $A$ is a localization of a countably generated R-algebra.

Proof. Let $d=\operatorname{dim} X$. By Katětov's theorem [17, Theorem 16.35, 16A, 16G] we can find $R_{0}=\mathbf{R}\left[\xi_{1}, \ldots, \xi_{d}\right] \subset B=\mathbf{R}^{X}$ such that the integral closure $R_{1}$ of $R_{0}$ in $B$ is dense in $B$. Note that the $\xi_{i}$ are algebraically independent otherwise we could apply the normalization lemma to get a similar $R_{0}^{\prime}$ with fewer generators. By $[17,16 \mathrm{G}], B$ is a separable metric space. Therefore so is $R_{1}$. Let $R \subset R_{1}$ be generated over $\mathbf{R}$ by a countable dense subset including $\xi_{1}, \ldots, \xi_{d}$. We now iterate the construction used in proving Theorem 4.1 a countable number of times using the fact that $K_{k}^{0}(X)$ is countable ( $k$ $=\mathbf{R}, \mathbf{C}, \mathbf{H})$ which is proved in $\$ 7$.

EXAMPLES. If $X$ is not triangulable, it will, in general, be impossible to find a noetherian ring $R \subset \mathbf{R}^{X}$ which is dense and has $\operatorname{dim} R=\operatorname{dim} X$. 
(1) Suppose $\operatorname{dim} R=0$. Then $R$ will have only a finite number of prime ideals. If $R$ is dense in $\mathbf{R}^{X}$, then $X \rightarrow \operatorname{spec} R$ by $x \mapsto\{f \in R \mid f(x)=0\}$ will be injective. Therefore any infinite 0 -dimensional space gives a counterexample.

(2) Let $X=X_{0} \cup X_{1} \cup \cdots \subset \mathbf{R}^{2}$ where $X_{0}=\{(0, y) \mid 0 \leqslant y \leqslant 1\}$ and $X_{n}$ $=\{(1 / n, y) \mid 0 \leqslant y \leqslant 1\}$. Suppose $R \subset \mathbf{R}^{X}$ is a 1-dimensional noetherian ring. If $P_{1}, \ldots, P_{r}$ are the minimal primes of $R$ and $f \in R-\cup P_{i}$, then $f$ lies in only a finite number of prime ideals. Therefore such an $f$ has only a finite number of zeros on $X$. Now, if $f$ changes sign on $X_{0}$, it will also change sign on each $X_{n}$ for large $n$ and so will have an infinite number of zeros. If $g \in R$ is not constant on $X_{0}$, we can find integers $m$ and $n$ such that $f=m+n g$ changes sign on $X_{0}$. For large integers $r, 1+r f$ will also change sign on $X_{0}$ and so $1+r f \in \cup P_{i}$. We can find $r \neq s$ with $1+r f, 1+s f \in P_{i}$. Therefore $(r-s) f$ $\in P_{i}$. Since $P_{i}$ is minimal, it consists of zero-divisors. It follows that no integer $\neq 0$ lies in $P_{i}$ and thus $f \in P_{i}$. Since $1+r f \in P_{i}$ we have a contradiction. This shows that all $g \in R$ are constant on $X_{0}$ so $R$ cannot be dense in $\mathbf{R}^{X}$.

7. Continuity. If $k=\mathbf{R}, \mathbf{C}$, or $\mathbf{H}$, we denote by $V B_{k}(X)$ the monoid of isomorphism classes of $k$-vector bundles on $X$ and by $K_{k}^{0}(X)$ the corresponding Grothendieck group. Special cases of the following continuity theorem were proved by Forster in [14].

THEOREM 7.1. Let $X=\operatorname{proj} \lim X_{\alpha}$ be a filtered inverse limit of compact spaces. Then $V B_{k}(X)=$ ind $\lim V B_{k}\left(X_{\alpha}\right)$ and $K_{k}^{0}(X)=\operatorname{ind} \lim K_{k}^{0}\left(X_{\alpha}\right)$.

It is very easy to deduce this from Theorem 3.5. We first need a rather trivial algebraic analogue :

LEMMA 7.2. Let $R=$ ind $\lim R_{\alpha}$ be a filtered direct limit of rings. Then $P(R)=$ ind $\lim P\left(R_{\alpha}\right)$ and $K_{0}(R)=$ ind $\lim K_{0}\left(R_{\alpha}\right)$.

This is, of course, very well known. To prove it we observe that any $P \in P(R)$ can be given as the image of an idempotent matrix $e \in M_{n}(R)$. Find a representative $e_{\alpha}$ for $e$ in $M_{n}\left(R_{\alpha}\right)$. Since $e^{2}=e$, we can find $\beta \geqslant \alpha$ such that the image $e_{\beta}$ of $e_{\alpha}$ in $M_{n}\left(R_{\beta}\right)$ satisfies $e_{\beta}^{2}=e_{\beta}$. Thus $P_{\beta}=\operatorname{im} e_{\beta} \in$ $P\left(R_{\beta}\right)$ and maps to $P$. This shows that ind $\lim P\left(R_{\alpha}\right) \rightarrow P(R)$ is onto. The injectivity is proved by a similar argument. We need only observe that if $e$ and $f$ are idempotent matrices, an isomorphism im $e \approx \operatorname{im} f$ can be described by a pair of matrices $\varphi, \psi$ with $\varphi=e \varphi=\varphi f, \psi=\psi e=f \psi, \varphi \psi=e, \psi \varphi=f$.

REMARK. Since any map ime $\rightarrow \operatorname{im} f$ can be described by a matrix $\psi$ with $\psi=\psi e=f \psi$, the same argument shows that we have an equivalence of categories $\mathscr{P}(R) \approx$ ind $\lim \mathscr{P}\left(R_{\alpha}\right)$ where $\mathscr{P}(R)$ is the category of finitely generated projective $R$-modules. However, in Theorem 7.1, the analogous statement is false in general. Let $\widetilde{T} G_{k}(X)$ be the category of $k$-vector bundles 
on $X$. The endomorphism ring of a trivial bundle in $\widetilde{T B}(X)$ is $k^{X}$. The corresponding ring in ind $\lim \mathcal{T}\left(X_{\alpha}\right)$ is ind $\lim k^{X_{\alpha}}$. In general these will be different. For example, if $X \subset \mathbf{R}^{m}$ and $\left\{X_{\alpha}\right\}$ is a base of neighborhoods of $X$, then ind $\lim k^{X_{\alpha}}$ consists of germs of functions defined on neighborhoods of $X$ and can be much bigger than $k^{X}$.

To prove Theorem 7.1, we let $B=\mathbf{R}^{X}, A_{\alpha}=\mathbf{R}^{X_{\alpha}}$, and $A=\operatorname{ind} \lim A_{\alpha}$. The projections $\pi_{\alpha}: X \rightarrow X_{\alpha}$ induce $\varphi_{\alpha}: A_{\alpha} \rightarrow B$ and hence $\varphi: A \rightarrow B$. I claim that Theorem 3.5 applies to $\varphi$. This will give $P\left(k \otimes_{\mathrm{R}} A\right) \approx P\left(k \otimes_{\mathrm{R}} B\right)$. Since $P\left(k \otimes_{\mathrm{R}} A\right)=$ ind $\lim P\left(k \otimes_{\mathrm{R}} A_{\alpha}\right)$ by Lemma 7.2, the required result follows.

We have only to check the conditions (1), (2), (3) and ( $\left.\mathrm{SBI}_{n}\right)(\mathrm{a})$. Clearly (1) is satisfied and (2) holds by the Stone-Weierstrass theorem. For (3), suppose $a \in A$ and $|\varphi(a)-1|<1$. Let $a_{\alpha} \in A_{\alpha}$ represent $a$ and let $U \subset X_{\alpha}$ be the open set defined by the condition $\left|a_{\alpha}-1\right|<1$. Then $\pi_{\alpha}: X \rightarrow X_{\alpha}$ has its image in $U$. By compactness, we can find $\beta \geqslant \alpha$ such that the projection $\pi_{\beta \alpha}: X_{\beta} \rightarrow X_{\alpha}$ has image in $U$. If $a_{\beta}$ is the image of $a_{\alpha}$ in $A_{\beta}$, it follows that $\left|a_{\beta}-1\right|<1$. Therefore $a_{\beta} \in A_{\beta}^{*}$ and so $a \in A^{*}$.

To check $\left(\operatorname{SBI}_{n}\right)(\mathrm{a})$, we use a similar argument. Let $z \in M_{n}(A)$ and suppose all entries of $\varphi(z) \in M_{n}(B)$ are less than $\delta$ in absolute value. By an argument analogous to the one just given, find a representative $z_{\beta} \in M_{n}\left(A_{\beta}\right)$ with all entries less than $\delta$ in absolute value. Let $f$ be as in the proof of Lemma 6.1. If $\delta$ is sufficiently small, $x_{\beta}=f \circ z_{\beta}$ will be defined and satisfy $x_{\beta}^{2}-x_{\beta}=z_{\beta}$. Furthermore, we can make all entries of $x_{\beta}$ less in absolute value than a prescribed $\varepsilon>0$ by taking $\delta$ sufficiently small. If $x \in M_{n}(A)$ is the image of $x_{\beta}$, then $x^{2}-x=z$ and $\varphi(x)$ has all entries less than $\varepsilon$ in absolute value.

CoRollary 7.3. If $X$ is a compact metric space, then $V B_{k}(X)$ and $K_{k}^{0}(X)$ are countable.

Proof. We can obtain $X$ as a countable filtered inverse limit of finite simplicial complexes, e.g. by modifying the procedure of [12, Chapter $X, \S 10]$ (or see below). Therefore, by Theorem 7.1 it is enough to treat the case where $X$ is a finite simplicial complex. In this case, the vector bundles on $X$ are classified by maps into Grassmann manifolds [41]. By triangulating these and applying the simplicial approximation theorem we see that only a countable number of homotopy classes are possible.

REMARK. Here is an alternative way to express $X$ as an inverse limit. Embed $X$ in the Hilbert cube $I^{\omega}$ [24]. Let $p_{n}: I^{\omega} \rightarrow I^{n}$ be projection on the first $n$ coordinates. Then $X=\operatorname{proj} \lim p_{n}(X)$. Therefore it will suffice to treat the case $X \subset \mathbf{R}^{n}, n<\infty$. In this case $X$ is a countable intersection of sets obtained by triangulating $\mathbf{R}^{n}$ and taking all simplexes which meet $X$.

8. The projective stable range. If $A$ is any ring, the projective stable range $\operatorname{psr} A$ is the least integer with the following property: If $P$ is a finitely generated 
projective $A$-module with rk $P \geqslant \operatorname{psr} A$ and if $(a, \pi) \in A \oplus P$ is unimodular, there is some $\eta \in P$ such that $\pi+a \eta$ is unimodular [44], [45]. The stable range sr $A$ is defined in the same way but with $P$ assumed free [44], [45], [46]. Therefore $\operatorname{sr} A \leqslant \operatorname{psr} A$. In [46], Vaserštein computed the stable range for rings of continuous functions. I will show that a minor modification of his argument enables us to compute the projective stable range.

As in [46], define $d(X)$ to be the largest integer $d$ such that there is a map $X \rightarrow \mathbf{R}^{d}$ with 0 as a stable value. If $X$ is normal, the argument of [24, Theorem VI 4] shows that $d(X)$ is characterized by the following property: $d(X)$ is the least integer such that if $n \geqslant d(X)$, any map of a closed subset of $X$ into $S^{n}$ can be extended to a map of $X$ into $S^{n}$. It follows immediately that $d(Y) \leqslant d(X)$ if $Y \subset X$ is closed. If $X$ is normal, $d(X)$ is equal to the covering dimension based on finite open coverings [34, Theorem 9.9]. If $X$ is paracompact this is the same as the covering dimension based on all open coverings [34, Corollary 9.14]. If $X$ is separable metric, it is well known that $d(X)$ $=\operatorname{dim} X[24]$.

THEOREM 8.1. Let $X$ be a paracompact Hausdorff space. Then

(1) $\mathrm{psr} \mathbf{R}^{X}=\operatorname{sr} \mathbf{R}^{X}=1+d(X)$,

(2) $\mathrm{psr} \mathbf{C}^{X}=\operatorname{sr} \mathbf{C}^{X}=1+\left[\frac{1}{2} d(X)\right]$,

(3) $\operatorname{psr} \mathbf{H}^{X}=\operatorname{sr} \mathbf{H}^{X}=1+\left[\frac{1}{4} d(X)\right]$.

The same applies to vector bundles on $X$. Thus the well-known stability theorems of Serre and Bass for vector bundles on finite complexes hold for paracompact spaces.

The calculation of $\operatorname{sr} k^{X}$ is due to Vaseršten [46]. Therefore, it will suffice to show that $\operatorname{psr} k^{X}$ is bounded by the numbers given at the right.

LEMMA 8.2. Let $X$ be a paracompact Hausdorff space and let $p: E \rightarrow X$ be $a$ fiber bundle over $X$ with fiber $S^{n}$. If $n \geqslant d(X)$, any section of $E$ over a closed subset of $X$ extends to $X$.

Proof. Let $s: Y \rightarrow E$ be a section over a closed set $Y \subset X$. Let $\left\{U_{\alpha}\right\}$ be a locally finite covering of $X$ such that $E \mid U_{\alpha}$ is trivial. Shrink $\left\{U_{\alpha}\right\}$ to a covering $\left\{V_{\alpha}\right\}$ with $\bar{V}_{\alpha} \subset U_{\alpha}$. Well-order the indices and let $X_{\alpha}=U_{\beta<\alpha} \bar{V}_{\beta}$. Suppose we can find sections $s_{\beta}$ of $p$ over $Y \cup X_{\beta}$ for $\beta<\alpha$ such that $s_{\beta}=s_{\gamma} \mid Y \cup X_{\gamma}$ for $\beta \leqslant \gamma$. If $\alpha$ is a limit ordinal, let $s_{\alpha}=\cup_{\beta<\alpha} s_{\beta}$. This is continuous because $\left\{Y, \bar{V}_{\beta}\right\}_{\beta<\alpha}$ is locally finite and $s_{\alpha}$ is continuous on each member of it. If $\alpha=\beta+1$, we can find a section $s^{\prime}$ over $\bar{V}_{\alpha}$ extending $s_{\beta} \mid \bar{V}_{\alpha} \cap\left(Y \cup X_{\beta}\right)$ because $E \mid \bar{V}_{\alpha}$ is trivial. Let $s_{\alpha} \mid Y \cup X_{\beta}=s_{\beta}$ and $s_{\alpha} \mid \bar{V}_{\alpha}=s^{\prime}$.

To prove Theorem 8.1, let $(a, \pi) \in A \oplus P$ be unimodular where $A=k^{X}, k$ $=\mathbf{R}, \mathbf{C}$, or $\mathbf{H}$. By [42, Theorem 2], $P$ can be identified with the module of sections of a vector bundle $\xi$ over $X$. Note that the compactness assumed in 
[42, Theorem 2] is not needed for this half of the theorem. Choose a metric on $\xi$ [42, Lemma 2] and let $S \rightarrow X$ be the sphere bundle $\{v \in \xi|| v \mid=1\}, D \rightarrow X$ the disc bundle $\{v \in \xi|| v \mid \leqslant 1\}$ and $D_{0} \rightarrow X$ the open disc bundle $\{v$ $\in \xi|| v \mid<1\}$.

Since $(a, \pi)$ is unimodular, $f(x)=|a(x)|+|\pi(x)|$ is never 0 on $X$. Therefore, $f$ is a unit of $k^{X}$. Replace $(a, \pi)$ by $\left(2 a f^{-1}, 2 \pi f^{-1}\right)$. In this way we can assume that $|a(x)|+|\pi(x)|=2$ for all $x \in X$.

Let $Y=\pi^{-1}(S)$ and extend $\pi \mid Y$ to a section $\tau$ of $S \rightarrow X$. This is possible provided the dimension of the fibre $|k: \mathbf{R}| \mathrm{rk} P-1$ is at least $d(X)$, i.e., $\operatorname{rk} P \geqslant 1+\left[|k: \mathbf{R}|^{-1} d(X)\right]$.

Define a section $\eta$ of $\xi$ by $\eta \mid X-\pi^{-1}\left(D_{0}\right)=0$ and $\eta \mid \pi^{-1}(D)$ $=a^{-1}(\tau|a|-\pi)$. Note that $|a|=1$ and $\tau=\pi$ on $\pi^{-1}(S)$, and $|a| \geqslant 1$ on $\pi^{-1}(D)$, so $\eta$ is well defined. Now $\pi+a \eta$ is $\pi$ on $X-\pi^{-1}\left(D_{0}\right)$ and $\tau|a| a^{-1}$ on $\pi^{-1}(D)$. Therefore, it is never zero and hence is unimodular. In fact, by [42, Proposition 1], if $\theta$ is the trivial line bundle, the map $\varphi: \theta \rightarrow \xi$ sending the unit section to $\pi+a \eta$ has image a subbundle of $\xi$. By [42, Proposition 2], $\varphi(\theta)$ is a direct summand of $\xi$. Therefore we get a map $\psi: \xi \rightarrow \theta$ taking $\pi+a \eta$ to the unit section. The induced map $P \rightarrow A$ takes $\pi+a \eta$ to 1 .

COROLlary 8.3. Let $X$ be a compact Hausdorff space and let $A \subset k^{X}, k$ $=\mathbf{R}, \mathbf{C}$, or $\mathbf{H}$ satisfy (2) and (3) of §2. Then $\operatorname{psr} A=\operatorname{sr} A=\operatorname{sr} k^{X}$.

Proof. By Theorem 2.2, $\operatorname{sr} A \leqslant \operatorname{psr} A \leqslant \operatorname{psr} k^{X}=\operatorname{sr} k^{X}$. The lower bound on $\operatorname{sr} A$ is the same by a result of Vaserštein [46].

9. Examples. I will now present some examples of rings having only trivial projective modules of low rank. All projective modules here are assumed to be finitely generated.

THEOREM 9.1. If $m \geqslant 2$ is an integer, there is a commutative noetherian ring A such that

(1) all projective A-modules of rank $<m$ are free,

(2) there is a projective A-module of rank $m$ which is not stably free.

The ring $A$ will be a $\mathbf{C}$-algebra of Krull dimension $2 m$. By using $\mathbf{R}$-algebras we can improve this to $2 m-2$ if $m$ is odd. It seems rather difficult to improve this any further by the present methods. In fact, by Corollary 8.3 no improvements will be possible for $\mathbf{C}$-algebras by the present methods because psr $A<m$ if $d(X)<2 m$.

THEOREM 9.2. If $m \geqslant 2$, there is a commutative noetherian ring $A$ such that

(1) all projective A-modules of rank $<m$ are free,

(2) there is a projective A-module of rank $m$ which is not free,

(3) all projective A-modules are stably free. 
In this theorem, $A$ will be a C-algebra of Krull dimension $2 m+2 p-3$ $\leqslant 6 m$ where $p$ is the least prime with $p>m$. This bound could presumably be improved in certain cases by using more detailed information about homotopy groups. If $m \equiv 2 \bmod 4$, a rather striking improvement is possible.

THEOREM 9.3. If $m \equiv 2 \bmod 4$, there is a commutative noetherian ring of Krull dimension $m$ such that

(1) A has a nonfree projective module of rank $m$,

(2) every projective module of rank $\neq m$ is free.

By a result of Bass [7, Corollary 4.2] such behavior is impossible for odd $m$. We would have to allow nontrivial projectives in at least two dimensions in this case. I do not know how to extend Theorem 9.3 to values of $m \neq \equiv 2 \bmod 4$.

By Lønsted's theorem, it will suffice to give examples of the required type for vector bundles over finite complexes. For Theorem 9.2 and 9.3 we can even take $A=R_{S}$ where $R$ is the ring of polynomial functions on $X$ since we are interested only in stably free modules. We could also use a localization of a polynomial ring as in $\$ 6$ at the expense of increasing the Krull dimension. This would have the advantage of producing a regular domain.

The complexes used will all be of the form $X=S^{d} \cup_{p} e^{d+1}$ obtained by attaching a $d+1$ cell to a $d$-sphere by a map $\partial e^{d+1} \rightarrow S^{d}$ of degree $p$.

As usual, $[X, Y]$ denotes the set of homotopy classes of maps from $X$ to $Y$.

LEMMA 9.4. Let $X$ be a CW complex and $Y$ a pathwise connected space. If $X$ or $Y$ is simply connected and $H^{n}\left(X, \pi_{n}(Y)\right)=0$ for all $n$, then $[X, Y]=0$.

Proof. This is a standard result of obstruction theory. If $X$ is finite, we can also use the following alternative argument which will be useful later. Suppose $Y$ is $d$-connected. If $d>\operatorname{dim} X$, the result is trivial. We use descending induction on $d$. Construct a fibration $Y^{\prime} \rightarrow Y \rightarrow^{p} K(\pi, d+1)$ where $Y^{\prime}$ is $(d+1)$-connected. If $f: X \rightarrow Y$ then $p f \sim 0$ since $H^{d+1}(X, \pi)=0$. By lifting this homotopy, $\operatorname{deform} f$ to a map $X \rightarrow Y^{\prime}$ and use induction. If $d=0, p f \sim 0$ because $X$ or $Y$ is simply connected so $p f$ lifts to a map of $X$ to the contractible universal covering of $K(\pi, 1)$.

LEMMA 9.5. Let $X$ be a $(d-2)$-connected finite $\mathrm{CW}$ complex with $d \geqslant 3$. Let $Y$ be a pathwise connected space. Let $f: X \rightarrow S^{d}$ and $g: S^{d} \rightarrow Y$. If $f^{*}[1] \otimes[g]$ $\neq 0$ in $H^{d}(X, \mathbf{Z}) \otimes \pi_{d}(Y)$, then $g f \neq 0$.

Proof. Suppose $Y$ is $(n-1)$-connected with $n \leqslant d-1$. As above, consider the fibration $Y^{\prime} \rightarrow Y \rightarrow^{p} K(\pi, n)$ with $Y^{\prime} n$-connected. Since $\pi_{d}\left(Y^{\prime}\right)$ $\rightarrow \approx \pi_{d}(Y)$, we can assume that $g: S^{d} \rightarrow Y^{\prime}$. If $g f \simeq 0$, then $g f$ extends to a map $h$ of the cone on $X, h: C X \rightarrow Y$. Since $g f(X) \subset Y^{\prime}$, ph factors as $C X \rightarrow S X \rightarrow^{k} K(\pi, n)$. Now $S X$ is $(d-1)$-connected and $d-1 \geqslant n$ so 
$H^{n}(S X, \pi)=0$. Therefore $k \simeq 0$. By lifting this homotopy, we deform $h$ rel $X$ to a map $C X \rightarrow Y^{\prime}$ and deduce that $g f \simeq 0$ as a map $X \rightarrow Y^{\prime}$. By repeating this process we reduce finally to the case where $Y$ is $(d-1)$-connected.

Consider again the fibration $Y^{\prime} \rightarrow Y \rightarrow^{p} K(\pi, d)$. It will suffice to show that $p g f \neq 0$. Therefore it is enough to do the case $Y=K(\pi, d)$. Let $\iota \in$ $H^{d}(Y, \pi)$ be the universal class. Then $g^{*}(\iota)=[g] \in H^{d}\left(S^{d}, \pi\right)=\pi$. We must show that $f^{*} g^{*}(\iota) \neq 0$. The universal coefficient theorem gives us a diagram

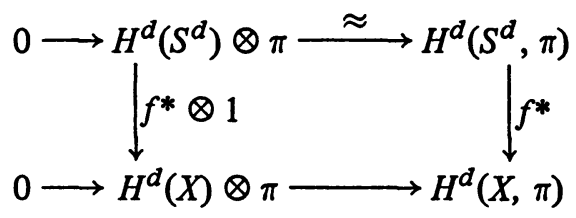

and $\left(f^{*} \otimes 1\right)(1 \otimes[g])=f^{*}(1) \otimes[g] \neq 0$.

CoROLlaRY 9.6. Let $X=S^{d-1} \cup_{p} e^{d}, d \geqslant 3$. Let $f$ be the canonical map $X \rightarrow X / S^{d-1}=S^{d}$. If $g: S^{d} \rightarrow Y$ with $[g] \notin p \pi_{d}(Y)$, then $g f \neq 0$.

In fact, $f^{*}(1)$ generates $H^{d}(X)=\mathbf{Z} / p \mathbf{Z}$ so $f^{*}(1) \otimes[g]$ is the image of $[g]$ in $H^{d}(X) \otimes \pi_{d}(Y)=\pi_{d}(Y) / p \pi_{d}(Y)$.

In proving the theorems, I will make use of the following standard result about the homotopy groups of the classical groups.

LEMMA 9.7. (a) $\pi_{i} B U(n)=\mathbf{Z} \oplus$ finite group if $i=2,4, \ldots, 2 n$ and is finite in all other cases.

(b) $\pi_{i} B S p(n)=\mathbf{Z} \oplus$ finite group if $i=4,8, \ldots, 4 n$ and is finite in all other cases.

(c) $\pi_{i} B O(2 n+1)=\mathbf{Z} \oplus$ finite group if $i=4,8, \ldots, 4 n$ and is finite for all other $i$.

(d) $\pi_{i} B O(4 n)=\mathbf{Z} \oplus$ finite group if $i=4,8, \ldots, 8 n-4, i \neq 4 n$, it is $\mathbf{Z} \oplus \mathbf{Z}$ $\oplus$ finite group if $i=4 n$, and is finite for all other $i$.

(e) $\pi_{i} B O(4 n+2)=\mathbf{Z} \oplus$ finite group if $i=4,8, \ldots, 8 n$ and if $i=4 n+2$. It is finite for all other $i$.

This is so well known that I was unable to find any reference where the author thought it necessary to write down the result explicitly. It is a special case of [40, Chapter V, §3]. It also follows immediately by induction using the fibrations

(a) $S^{2 n-1} \rightarrow B U(n-1) \rightarrow B U(n)$,

(b) $S^{4 n-1} \rightarrow B S p(n-1) \rightarrow B S p(n)$,

(c) $V \rightarrow B O(2 n-1) \rightarrow B O(2 n+1)$,

(d, e) $S^{2 n-1} \rightarrow B O(2 n-1) \rightarrow B O(2 n)$,

where $V$ in (c) is the Stiefel manifold $V=O(2 n+1) / O(2 n-1)$. We use the homotopy sequences for these fibrations and the fact that $\pi_{m} S^{n}$ is finite for $n$ 
odd, $n \neq m$ [23, Theorem 7.1] and $\pi_{n} S^{n}=\mathbf{Z}$. For (c) use similar results for $V$ given in $[23, \S 11]$. These same fibrations give us the well-known stability theorems $\pi_{i} B U(n) \rightarrow \approx \pi_{i} B U$ for $i \leqslant 2 n, \pi_{i} B S p(n) \rightarrow \approx \pi_{i} B S p$ for $i \leqslant 4 n+2$, $\pi_{i} B O(n) \rightarrow \approx \pi_{i} B O$ for $i \leqslant n-1$. Recall also that $\pi_{i} B U=\mathbf{Z}$ for $i$ even and positive and $\pi_{i} B U=0$ for $i$ odd [8].

To prove Theorem 9.1, choose $X=S^{2 m-1} \cup_{p} e^{2 m}$ where $p$ is a prime which does not divide the orders of the finite groups $\pi_{2 m} B U(n), \pi_{2 m-1} B U(n)$ for $n<m$. By Lemma 9.4, $[X, B U(n)]=0$ for $n<m$ so all vector bundles on $X$ of rank $<m$ are trivial. Let $g: S^{2 m} \rightarrow B U(m)$ generate $\pi_{2 m} B U(m)=\mathbf{Z}$. Then gf: $X \rightarrow B U(m)$, with $f$ as in Corollary 9.6, defines a vector bundle $\xi$ on $X$ of rank $m$. The composition $g^{\prime}: S^{2 m} \rightarrow^{g} B U(m) \rightarrow B U$ generates $\pi_{2 m} B U=\mathbf{Z}$. By Corollary $9.6 g^{\prime} f \neq 0$ so $\xi$ is stably nontrivial.

If $m \geqslant 3$ is odd, a similar argument works using $B O(n)$ and $X=S^{2 m-3}$ $\cup_{p} e^{2 m-2}$.

To prove Theorem 9.3, we use the fact that for $m \equiv 2 \bmod 4, \pi_{m} B O(n)$ is finite for $n \neq m$ while $\pi_{m} B O(m)=\mathbf{Z} \oplus$ finite group. Also $\pi_{m-1} B O(n)$ is always finite. Since $\pi_{i} B O(n) \rightarrow \approx \pi_{i} B O$ for $n>i$, only a finite number of distinct finite groups occur among the $\pi_{m} B O(n)$ and $\pi_{m-1} B O(n)$. Let $p$ be a prime which does not divide the orders of any of these groups and let $X=S^{m-1} \cup_{p} e^{m}$. Lemma 9.4 shows that $[X, B O(n)]=0$ for $n \neq m$. If $g: S^{m} \rightarrow B O(m)$ generates a summand $\mathbf{Z}$ of $\pi_{m} B O /(m)$, then $g f \neq 0$ by Corollary 9.6.

To prove Theorem 9.2, it will suffice to find an odd $i$ and a prime $p$ such that $\pi_{i} B U(n)$ and $\pi_{i-1} B U(n)$ are finite of order prime to $p$ for $n<m$ while $\pi_{i} B U(m)$ has a nontrivial $p$-component.

Suppose we have such an $i$ and $p$. Let $X=S^{i-1} \cup_{p} e^{i}$. As above, we get $[X, B U(n)]=0$ for $n<m$ while $[X, B U(m)] \neq 0$. By [4] or [11] there is a spectral sequence $H^{r}\left(X, K^{s}(\mathrm{pt})\right) \Rightarrow K^{*}(X)$. Now $K^{s}(\mathrm{pt})$ is 0 for $s$ odd and $\mathbf{Z}$ for $s$ even, while $H^{r}(X, \mathbf{Z})=0$ for $r$ even because $i$ is odd. Therefore, the $E_{2}$ term has ncthing in dimension 0 except $E_{2}^{00}=\mathbf{Z}$. Therefore, $K^{0}(X)=\mathbf{Z}$ and all complex vector bundles on $X$ are stably trivial.

The following lemma shows that we can choose $p$ to be any prime $>m$ and $i=2 m+2 p-3$.

LEMMA 9.8. If $p$ is a prime with $p>m \geqslant 2$, then

(a) $\pi_{i} B U(n)$ is finite of order prime to $p$ for $n<m$ and $i=2 m+2 p-4$ or $i=2 m+2 p-3$.

(b) $\pi_{2 m+2 p-3} B U(m)$ has nontrivial p-torsion.

To prove this, we first quote a result on homotopy groups of spheres.

Lemma 9.9. Let $p$ be an odd prime. Then $\pi_{i}\left(S^{2 n-1}\right)$ has p-torsion for $i=2 n+2 p-4$ but for no other $i \leqslant 2 n+4 p-7$. 
See [23, Chapter XI, Corollaries 9.3, 10.5].

To prove Lemma 9.8, consider the fibration

This gives

$$
S^{2 n-1}=U(n) / U(n-1) \rightarrow B U(n-1) \rightarrow B U(n) .
$$

$$
\pi_{i} S^{2 n-1} \rightarrow \pi_{i} B U(n-1) \rightarrow \pi_{i} B U(n) \rightarrow \pi_{i-1} S^{n-1} .
$$

Lemma 9.8(a) follows from this by induction using Lemma 9.9. In the trivial case $n=1$, only $\pi_{2} B U(1)$ is nonzero.

For (b), set $i=2 m+2 p-3$ in

$$
\pi_{i} B U(m-1) \rightarrow \pi_{i} B U(m) \rightarrow \pi_{i-1} S^{2 m-1} \rightarrow \pi_{i-1} B U(m-1) .
$$

The end terms have no $p$-torsion by (a) while $\pi_{i-1} S^{2 m-1}$ has $p$-torsion by Lemma 9.9. Since $\pi_{i} B U(m)$ is finite, it must have $p$-torsion.

10. Nonvanishing polynomials. I will conclude by discussing some questions related to Hilbert's 17 th problem suggested by the above results. The basic problem is to give a purely algebraic characterization of the rings arising from the construction of $\$ 6$. In general, this seems too messy to be worth pursuing. I will, therefore, consider only the special case in which $R$ is the ring of polynomial functions on a set $X \subset \mathbf{R}^{n}, S$ is the set of such functions with no zeros on $X$, and $A=R_{S}$. This will suffice for the rings needed in Theorems 9.2 and 9.3 and for the example of $S^{n-1}$ considered in $\$ 11$. There is usually little trouble in describing $R$ algebraically. The problem is to characterize $S$.

Let us assume that $X$ is defined by polynomial equations and inequalities $f_{i}(x)=0, g_{j}(x) \geqslant 0$. A polynomial $h$ will have no zeros on $X$ if and only if the conditions $f_{i}=0, g_{j} \geqslant 0$ imply $h^{2}>0$. Since the theory of elementary algebra over $\mathbf{R}$ is decidable [36], [37] there is an algorithm which enables us to check whether this condition is fulfilled. Therefore, given $h$, we can decide whether $h \in S$. This gives a solution to our problem but it would be nice to have a simpler and more explicit one.

An approximation to $S$ can be obtained as follows. If there are no relations $g_{j} \geqslant 0$ we set $\Sigma=\left\{1+\sum a_{i}^{2} \mid a_{i} \in R\right\}$. If there is a single relation $g \geqslant 0$, set $\Sigma=\left\{1+\sum a_{i}^{2}+g \sum b_{j}^{2} \mid a_{i}, b_{j} \in R\right\}$. In general, suppose we have $r$ relations $g_{j} \geqslant 0, j=1, \ldots, r$. For each subset $J$ of $\{1, \ldots, r\}$ set $g_{(J)}=\prod g_{j}$ over $j \in J$ and let $g_{(\varnothing)}=1$. We then take $\Sigma$ to be the set of all $1+\sum_{J} \Sigma_{k} b_{J k}^{2} g_{(J)}$ with $b_{J k} \in R$.

If $X=\mathbf{R}^{n}$, examples due to Hilbert [21] and others [32], [38] show that $\Sigma \neq S$. It is conceivable that $\Sigma$ might equal $S$ if $X$ is compact. Some evidence in this direction is given by $[38, \S 3]$. However, for our purposes $\Sigma$ is already big enough.

THEOREM $10.1 \mathrm{~A}=R_{S}=R_{\Sigma}$. 
This is equivalent to the assertion that every element of $S$ divides an element of $\Sigma$. The fact that $A=R_{\Sigma}$ gives us the required algebraic description of $A$.

In proving Theorem 10.1, I will make use of the following extension of $A$. Robinson's generalization of Hilbert's 17th problem [37].

THEOREM 10.2. Let $Y \subset \mathbf{R}^{n}$ be an irreducible real algebraic variety and let $R$ be the ring of polynomial functions on $Y$. Let $g_{1}, \ldots, g_{r} \in R$ and assume that for each $y \in Y$ there is an $i$ with $g_{i}(y) \leqslant 0$. Then there is a nontrivial relation $\Sigma_{J} \sum_{k} c_{J, k}^{2} g_{(J)}=0$ in $R$.

Here $g_{(J)}=\prod_{i \in J} g_{i}$ as above. Nontriviality simply means that some $c_{J, k} \neq 0$. In Robinson's theorem $r=1$ and it follows that there is a relation $\sum a_{i}^{2}+b^{2} g=0$ where $b=\sum c_{(1), k}^{2} \neq 0$.

I will give a proof similar to that of Robinson's theorem in [19]. We can assume that no $g_{i}$ is identically zero, otherwise $g_{i}=0$ is the required relation. Let $Y$ be defined by equations $f_{j}(x)=0$ and set $f=\sum f_{j}^{2}$ so that $Y$ $=\{y \mid f(y)=0\}$. Consider the elementary statement

$$
(\forall x)\left[(f(x)=0) \supset\left(\tilde{g}_{1}(x) \leqslant 0\right) \wedge \cdots \wedge\left(\tilde{g}_{r}(x) \leqslant 0\right)\right],
$$

where $\tilde{g}_{i}$ is a polynomial representing $g_{i} \in R$. This holds for $\mathbf{R}$ and hence, by the completeness of the theory of real-closed fields [36], [37], it holds for any real-closed field containing $\mathbf{R}$. Therefore, it holds for any ordered field containing $\mathbf{R}$ since such a field can be completed to a real closed field and no existential quantifiers are involved. Let $K$ be the quotient field of $R$. It is formally real since $\sum\left(h_{i} / h\right)^{2}=0$ in $K$ implies $\sum h_{i}^{2}=0$ in $R$ and hence all $h_{i}=0$. Let $\xi_{i}$ be the image in $R \subset K$ of the coordinate function $x_{i}$. Then $f(\xi)=0$. Therefore, by $(*)$, we have $g_{i}=\tilde{g}_{i}(\xi) \leqslant 0$ for some $i$ in each ordering of $K$. Since $g_{i} \neq 0$ in $R$, we even have $g_{i}<0$. Therefore the theorem follows from the next lemma which is based on an argument of Artin [1].

LEMMA 10.3. Let $K$ be a formally real field and let $g_{1}, \ldots, g_{r} \in K$. Assume that in each ordering of $K$ we have $g_{i}<0$ for some $i$. Then there is a nontrivial relation $\Sigma_{J} \Sigma_{k} c_{J, k}^{2} g_{(J)}=0$ in $K$.

Proof. Suppose the conclusion is false and let $L \supset K$ be an algebraic extension which is maximal with respect to the property that no relation of the required form holds in $L$. Then $L$ is formally real since if $\sum e_{i}^{2}=0$ in $L$, then $\sum e_{i}^{2} g_{(\varnothing)}=0$ would contradict the definition of $L$.

Suppose $\sqrt{g_{i}} \notin L$ for some $i$. Then in $L\left(\sqrt{g_{i}}\right)$ there will be a nontrivial relation

$$
\sum_{J, k}\left(a_{J, k}+\sqrt{g_{i}} b_{J, k}\right)^{2} g_{(J)}=0
$$


where $a_{J, k}, b_{J, k} \in L$. This gives us

$\sum\left(a_{J, k}^{2}+g_{i} b_{J, k}^{2}\right) g_{(J)}=\sum a_{J, k}^{2} g_{(J)}+\sum_{i \notin J} b_{J, k}^{2} g_{(J \cup\{i\})}+\sum_{i \in J} b_{J, k}^{2} g_{i}^{2} g_{(J-\{i\})}=0$

which is a nontrivial relation over $L$. It follows that $\sqrt{g_{i}} \in L$ for all $i$. Now choose an ordering for $L$. Since $\sqrt{g_{i}} \in L$, we have $g_{i} \geqslant 0$ for all $i$. Therefore, the induced ordering on $K$ contradicts our hypothesis.

We will also need one of the basic results of Dubois and Efroymson [10]. In their terminology, a ring $R$ is called real if $\sum a_{i}^{2}=0$ in $R$ implies all $a_{i}=0$. An ideal $I$ of such a ring is called real if $R / I$ is real, i.e. if $\sum a_{i}^{2} \in I$ implies all $a_{i} \in I$. If $I \subset \mathbf{R}\left[x_{1}, \ldots, x_{n}\right]$, let $V(I)=\left\{\xi \in \mathbf{R}^{n} \mid f(\xi)=0\right.$ for all $\left.f \in I\right\}$. If $X \subset \mathbf{R}^{n}$, let $g(X)=\left\{f \in \mathbf{R}\left[x_{1}, \ldots, x_{n}\right] \mid f(\xi)=0\right.$ for all $\left.\xi \in X\right\}$.

THEOREM 19.4 [10]. Let $P$ be a real prime ideal of $\mathbf{R}\left[x_{1}, \ldots, x_{n}\right]$. Then $g(V(P))=P$.

Here is a simple proof based on the idea of [19] used above. Let $R=\mathbf{R}\left[x_{1}, \ldots, x_{n}\right] / P$ and let $K$ be the quotient field of $R$. Since $R$ is real by hypothesis, $K$ is a formally real field as observed above. Suppose $g$ $\in g(V(P))$. Let $f_{1}, \ldots, f_{n}$ generate $P$, set $f=\sum f_{i}^{2}$, and consider the elementary statement

$$
(\forall x)[(f(x)=0) \supset(g(x)=0)] .
$$

This holds for $\mathbf{R}$ and hence, by the argument used above, it holds for all formally real fields containing $\mathbf{R}$ and, in particular, for $K$. Let $\xi_{i}$ be the image in $K$ of the coordinate function $x_{i}$. Then $f(\xi)=0$ so $g(\xi)=0$ but this means that $g \in P$.

A number of fundamental results of [10] can be easily deduced from this.

COROLlaRY 10.5. Let $I$ be any ideal of $\mathbf{R}\left[x_{1}, \ldots, x_{n}\right]$.

(a) $\mathscr{G}(V(I))$ is the intersection of all real prime ideals containing $I$.

(b) $f \in g(V(I))$ if and only if $f^{2 n}+\sum g_{i}^{2} \in I$ for some $n$ and

$$
g_{i} \in \mathbf{R}\left[x_{1}, \ldots, x_{n}\right] \text {. }
$$

(c) $I=9(V(I))$ if and only if $I$ is real.

Note that (b) is a slight refinement of the "Reelnullstellensatz" of [10].

Proof. It is clear that (b) implies (c) since any ideal of the form $g(X)$ is real. To prove (a) and (b) we use the following lemma which is based on the argument of [10, Lemma, p. 112].

LEMMA 10.6. Let $R$ be a real ring and $S$ a multiplicatively closed subset of $R$ such that $s \in S, a \in R$ imply $s^{2}+a^{2} \in S$. If $P$ is an ideal maximal with respect to $P \cap S=\varnothing$ then $P$ is a real prime ideal. 
Proof. It is a standard fact that $P$ is a prime ideal. Suppose $\sum_{1}^{n} a_{i}^{2} \in P$ but $a_{1} \notin P$. Then $\left(P, a_{1}\right) \cap S \neq \varnothing$ so we have $r a_{1} \equiv s \bmod P$ with $s \in S$. Therefore $r^{2} a_{1}^{2} \equiv s^{2} \bmod P$ and so $s^{2}+\sum_{2}^{n}\left(r a_{i}\right)^{2} \equiv r^{2} \sum a_{i}^{2} \equiv 0 \bmod P$ but this element is in $S$.

Now let $S=\left\{f^{2 n}+\sum g_{i}^{2}\right\}$. We must show that $f \in \mathscr{g} V(I)$ if and only if

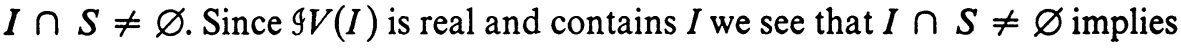
$f \in g V(I)$. Suppose then that $I \cap S=\varnothing$. Let $P \supset I$ be an ideal maximal with respect to $P \cap S=\varnothing$. By Lemma $10.6, P$ is a real prime ideal. Therefore, by Theorem 10.4, $P=\mathscr{G V}(P) \supset \mathscr{G}(I)$. Thus $S \cap \mathscr{}(I)=\varnothing$ and so $f \notin \mathscr{G}(I)$. Since $f \notin P$, this also implies (a).

To prove Theorem 10.1 we will also need the following generalization of Lemma 10.6.

LEMMA 10.7. Let $\Sigma$ be as in Theorem 10.1 Let $P$ be an ideal maximal with respect to $P \cap \Sigma=\varnothing$. Then $\Sigma b_{J, k}^{2} g_{(J)} \in P$ implies $b_{J, k} g_{(J)} \in P$ for all $J$ and $k$. In particular, $P$ is real.

Proof. By the maximality of $P$ we need only show $\left(P, b_{J, k} g_{(J)}\right) \cap \Sigma=\varnothing$. If not, we can find $r b_{J, k} g_{(J)} \equiv \sigma \bmod P$ with $\sigma \in \Sigma$. Therefore $\tau=\sigma^{2}+$ $r^{2} g_{(J)} \dot{\Sigma}^{\prime} b_{I, l}^{2} g_{(I)} \equiv 0 \bmod P$ where $\Sigma^{\prime}$ is taken over all indices except $J, k$, but $\tau$ clearly lies in $\Sigma$.

We can now prove Theorem 10.1. Suppose $s \in S$ divides no element of $\Sigma$. Then $(s) \cap \Sigma=\varnothing$. Let $P \supset(s)$ be an ideal maximal with respect to $P \cap \Sigma$ $=\varnothing$. Then $P$ is a real prime ideal by Lemma 10.7. Let $P_{1} \subset \mathbf{R}\left[x_{1}, \ldots, x_{n}\right]$ be the inverse image of $P$. It is real so by Theorem $10.4, P_{1}=g(Y)$ where $Y=V\left(P_{1}\right)$ is an irreducible real algebraic variety. Since $s \in P$ and $s$ is never zero on $X$, we have $X \cap Y=\varnothing$. Now $f_{i} \in \operatorname{ker}\left[\mathbf{R}\left[x_{1}, \ldots, x_{n}\right] \rightarrow R\right]$ so $f_{i} \in$ $P_{1}$. Since $X \cap Y=\varnothing$ we see that for each $y \in Y$ we must have $g_{j}(y)<0$ for some $j$. Suppose $j=1, \ldots, s$ are the indices for which this can occur. Theorem 10.2 now gives us a nontrivial relation $\sum c_{J, k}^{2} g_{(J)} \equiv 0$ in $R / P$, where $J \subset\{1, \ldots, s\}$. By Lemma 10.7 we have $c_{J, k} g_{(J)} \in P$ for all $J$ and $k$ but $g_{(J)} \notin P$ since $J \subset\{1, \ldots, s\}$ and $g_{j}$ is not zero on $Y$ for $1 \leqslant j \leqslant s$. Therefore $c_{J, k} \in P$ for all $J, k$ and our relation in $R / P$ is trivial after all.

As a consequence of Theorem 10.1 we get a special case of a result of Hörmander [22].

CoROllary 10.8. Let $f$ be a polynomial such that $f(x) \neq 0$ for $x \in \mathbf{R}^{n},|x|$ $\geqslant R$. Then we have $|f(x)| \geqslant C\left(1+|x|^{2}\right)^{-N}$ for $|x| \geqslant R$.

Hörmander's estimate is, of course, much more precise in the neighborhood of $\{f=0\}$. To prove Corollary 10.8, take $g=|x|^{2}-R^{2}$ in Theorem 10.1 and get $f h=1+\sum a_{i}^{2}+\sum b_{j}^{2} g$ so $f h \geqslant 1$ for $|x| \geqslant R$. Then let $2 N$ be $\geqslant \operatorname{deg} h$.

Gorin [18] has pointed out that even if $f(x)>0$ for all $x \in \mathbf{R}^{n}$ we can still 
have $\inf f=0$ e.g. let $f(x, y)=(x y-1)^{2}+y^{2}$ on $\mathbf{R}^{2}$ and set $y=x^{-1}$. It would be interesting to know if, in case $f(x)>0$ for all $x$, one can find a bound for $N$ in terms of $\operatorname{deg} f$ and $n$.

The argument above can also be used to prove an interesting extension theorem

COROLlary 10.9. Let $X$ be a real algebraic variety and let $\varphi$ be a polynomial function such that $\varphi(x)>0$ for all $x \in X$. Then there is a polynomial $g$ representing $\varphi$ such that $g(x)>0$ for all $x \in \mathbf{R}^{n}$.

Proof. Choose any polynomial $h$ representing $\varphi$ and let $Y=\{h \leqslant 0\}$. Then $X \cap Y=\varnothing$. If $X=\{f=0\}$ then $f$ is never zero on $Y$. By Theorem 10.1 we can write $f(y) k(y)=1+\sum a_{i}(y)^{2}$ for $y \in Y$. If $2 N>\operatorname{deg} k$, it follows as above that $|f(y)| \geqslant C\left(1+|y|^{2}\right)^{-N}$ for $y \in Y$. Let $g(x)=h(x)$ $+A\left(1+|x|^{2}\right)^{M+N} f(x)^{2}$. If $h(x)>0$ then $g(x)>0$. If $h(x) \leqslant 0$ then $x \in Y$ so $g(x) \geqslant h(x)+A C\left(1+|x|^{2}\right)^{M}>0$ if $2 M>\operatorname{deg} h$ and $A$ is sufficiently large.

EXAMPLE. In [19] it is shown that if $\varphi \geqslant 0$ on $X$ it is not generally possible to find a polynomial $\psi$ representing $\varphi$ with $\psi \geqslant 0$ on $\mathbf{R}$. The example in [19] was not compact but can easily be modified as follows. Let $X \subset \mathbf{R}^{2}$ be defined by $f=x^{4}-x^{3}+y^{2}=0$. This $X$ is compact and homeomorphic to a circle. Let $\varphi(x, y)=x$. Then $\varphi \geqslant 0$ on $X$. If $\psi \mid X=\varphi$, Lemma 10.10 below and the irreducibility of $f$ shows that $\psi=x+\left(x^{4}-x^{3}+y^{2}\right) g(x, y)$. Therefore $\psi(x, 0)$ $=x+O\left(x^{3}\right)$ and is negative for $x<0$ and near zero. As in [19] it follows that $\varphi$ cannot be a sum of squares of elements of $R$.

Lemma 10.10. Let $X \subset \mathbf{R}^{n}$ be defined by an irreducible polynomial equation $f(x)=0$. If the projection of $X$ on some hyperplane contains a nonempty open set, then the ideal of polynomials zero on $X$ is generated by $f$.

Proof. Suppose the image of $X$ in $\mathbf{R}^{n-1}$ under the map $\left(x_{1}, \ldots, x_{n}\right)$ $\mapsto\left(x_{1}, \ldots, x_{n-1}\right)$ contains a nonempty open set $U$. Let $g$ be a polynomial which is zero on $X$. If $f \nmid g$ then $f \nmid g$ in the ring $R=\mathbf{R}\left(x_{1}, \ldots, x_{n-1}\right)\left[x_{n}\right]$ otherwise we would get $g s=f h$ in $\mathbf{R}\left[x_{1}, \ldots, x_{n}\right]$ with $s \in \mathbf{R}\left[x_{1}, \ldots, x_{n-1}\right]$. If $f \nmid g$ then $f \mid s$ since $f$ is irreducible. Therefore $f \in \mathbf{R}\left[x_{1}, \ldots, x_{n-1}\right]$. But then $f \mid U=0$ so $f=0, X=\mathbf{R}^{n}$, and $g=0$. Now if $f \nmid g$ in $R$ we have $R f+R g$ $=R$ since $f$ remains irreducible in $R$. Write $p f+q g=s$ in $\mathbf{R}\left[x_{1}, \ldots, x_{n}\right]$ with $s \in \mathbf{R}\left[x_{1}, \ldots, x_{n-1}\right]$. If $x^{\prime}=\left(x_{1}, \ldots, x_{n-1}\right) \in U$, find $x=\left(x_{1}, \ldots, x_{n}\right) \in X$. Since $f(x)=g(x)=0$ we get $s\left(x^{\prime}\right)=0$ for $x^{\prime} \in U$. Therefore $s=0$, a contradiction.

REMARK. The hypothesis of the lemma is clearly satisfied if $\operatorname{grad} f \neq 0$ at some point of $X$. In fact, Whitney's stratification theorem [47] shows that both these conditions are equivalent to $\operatorname{dim} X=n-1$. The example $f=x^{2}+y^{2}$ 
shows that some such condition is needed.

If $\operatorname{grad} f \neq 0$ at all points of $X$ then $X$ is an analytic submanifold. If $f=g h$ then $\operatorname{grad} f=0$ at all points where $g=h=0$. Therefore, if $X$ is connected, $f=p h$ where $p$ is irreducible and $h$ is never 0. Note $\operatorname{grad} f=h \operatorname{grad} p$ on $X$. The lemma shows that $p$ generates the ideal of polynomials zero on $X$.

For sets $X$ of the sort considered in Theorem 10.1, I have only been able to prove an analogue of Corollary 10.9 under a rather peculiar hypothesis.

THEOREM 10.11. Let $X \subset \mathbf{R}^{n}$ be defined by polynomial relations $f=0, g_{i}$ $\geqslant 0, i=1, \ldots, r$. Suppose there are polynomials $c_{i j}$,d such that $\sum c_{i j}(x)^{2} g_{j}(x)$ $+d f<0$ for large $|x|$. Let $\varphi$ be a polynomial with $\varphi(x)>0$ for all $x \in X$. Then there are polynomials $h, a_{i j}$, b such that $h(x) \geqslant \varepsilon>0$ for all $x \in \mathbf{R}^{n}$ and such that $\varphi=h+\sum a_{i j}^{2} g_{j}+b f$.

Note that the hypothesis implies that $X$ is compact. Possibly it holds whenever $X$ is compact. In any case, we can force it to hold for compact $X$ by adding one more relation $g_{0}(x)=R^{2}-|x|^{2} \geqslant 0$ to the definition of $X$.

EXAMPLE. The theorem is false if $X$ is not compact. Let $X \subset \mathbf{R}^{2}$ be defined by $x y^{2} \geqslant 1$ and let $\varphi(x, y)=x$. Then $\varphi>0$ on $X$ but no $h(x, y)=x$ $-\sum a_{i}(x, y)^{2}\left(x y^{2}-1\right)$ can have $h>0$ on all $\mathbf{R}^{2}$. If $h>0$, then

$$
0 \leqslant \sum a_{i}(x, y)^{2}<x\left(x y^{2}-1\right)^{-1} \quad \text { for }(x, y) \in X .
$$

Therefore, if $x>0$ is fixed, we get $\lim _{y \rightarrow \infty} \sum a_{i}(x, y)=0$. Since we are dealing with polynomials in $y$, it follows that $a_{i}(x, y)=0$ for each fixed $x>0$ and so $a_{i}=0$.

I will prove the theorem in two steps, first extending to a ball and then to all of $\mathbf{R}^{n}$.

TheORem 10.12. Let $X \subset \mathbf{R}^{n}$ be defined by polynomial relations $f=0, g_{i}$ $\geqslant 0, i=1, \ldots, r$. Assume that $X$ lies in the ball $B=\{x|| x \mid \leqslant R\}$. Then $a$ polynomial $\varphi(x)$ satisfies $\varphi(x)>0$ for all $x \in X$ if and only if it can be expressed in the form

$$
\varphi=\psi+\sum a_{i j}^{2} g_{j}+b f
$$

where $\psi, a_{i j}, b \in \mathbf{R}\left[x_{1}, \ldots, x_{n}\right]$ and $\psi(x)>0$ for all $x \in B$.

Proof. We can replace $f$ by $f^{2}$ and so assume that $f(x) \geqslant 0$ for all $x$. By using $\alpha g_{i}$ for $g_{i}, \alpha>0$, we can assume that $\left|g_{i}(x)\right| \leqslant 1$ for $x \in B$. Suppose $\varphi(x) \geqslant \varepsilon>0$ for $x \in X$. Let $U=\{x \mid \varphi(x)>\varepsilon / 2\}$. For $a>0$ define $X_{a}$ $=\left\{x \in B \mid f(x) \leqslant a, g_{i}(x) \geqslant-a\right\}$. Then $X=\cap X_{a}$. Since $B$ is compact, there is some $a>0$ with $X_{a} \subset U$. Now define

$$
\psi=\varphi-\eta^{2} \sum_{1}^{r}\left(g_{i}-1\right)^{2 n} g_{i}+\eta^{2}(1+f)^{2 n} f
$$


where $\eta \in \mathbf{R}$. Since $\left|g_{i}\right| \leqslant 1$ on $B$ we see that $\left|\left(g_{i}-1\right)^{2 n} g_{i}\right| \leqslant 1$ if $g_{i} \geqslant 0$. The terms with $g_{i} \leqslant 0$ and the term in $f$ are always nonnegative. Therefore, if $x \in U$, we have $\psi(x) \geqslant \varepsilon / 2-r \eta^{2}>0$ if $\eta$ is sufficiently small. Suppose $|\varphi(x)| \leqslant A$ for $x \in B$. If $x \in B-U$ we have either $f(x)>a$ or $g_{i}(x)<-a$ for some $i$. Therefore $\psi(x) \geqslant-A-r \eta^{2}+\eta^{2} a(1+a)^{2 M}>0$ if $M$ is suffciently large.

THEOREM 10.13. Let $\psi$ and $\Phi$ be polynomials on $\mathbf{R}^{n}$. Suppose $\psi(x)>0$ for $|x| \leqslant R$ and $\Phi(x)>0$ for $|x| \geqslant R$. Then for suitable $\delta \neq 0, \eta \in \mathbf{R}$ and $M$ we have

$$
\psi(x)+\eta^{2}|x|^{2 M} \Phi(x) \geqslant \delta>0
$$

for all $x \in \mathbf{R}^{n}$.

Proof. By Corollary $10.8,|\Phi(x)||x|^{2 N} \rightarrow \infty$ as $|x| \rightarrow \infty$ if $N$ is sufficiently large. After replacing $\Phi$ by $|x|^{2 N} \Phi(x)$, we can assume that $\Phi(x) \geqslant a>0$ for $|x| \geqslant R$.

Let $\psi(x) \geqslant \varepsilon>0$ for $|x| \leqslant R$. For some $r>0$ we will have $\psi(x) \geqslant \varepsilon / 2$ for $|x| \leqslant R+r$. Let $F(x)=\psi(x)+\rho^{2}\left(|x| R^{-1}\right)^{2 M} \Phi(x)$. Suppose $|\Phi(x)| \leqslant A$ for $|x| \leqslant R$. If $|x| \leqslant R$, then $F(x) \geqslant \varepsilon-\rho^{2} A>0$ for small $\rho$. If $R \leqslant|x|$ $\leqslant R+r$, then $F(x) \geqslant \varepsilon / 2$ since $\Phi(x)>0$. If $|x| \geqslant R+r$, then $|\psi(x)|$ $\leqslant C|x|^{d}$ where $d=\operatorname{deg} \psi$. Therefore

$$
\begin{aligned}
F(x) & \geqslant-C|x|^{d}+\rho^{2}\left(|x| R^{-1}\right)^{2 M} a=|x|^{d}\left[-C+\rho^{2}|x|^{2 M-d} R^{-2 M} a\right] \\
& \geqslant|x|^{d}\left[-C+\rho^{2}\left(1+r R^{-1}\right)^{2 M-d} R^{d} a\right]>0
\end{aligned}
$$

if $M$ is sufficiently large.

Now, to prove Theorem 10.11 , let $\Phi=-\sum c_{i j}^{2} g_{j}-d f$ and choose $R$ so that $\Phi(x)>0$ for $|x|>R$. Then $X \subset B=\{x|| x \mid \leqslant R\}$. Write $\varphi$ in the form (*) of Theorem 10.12. By Theorem 10.13 we get $\psi_{1}=\psi+\eta^{2}|x|^{2 M} \Phi \geqslant \delta>0$ on $\mathbf{R}^{n}$ and $\varphi=\psi_{1}+\eta^{2}|x|^{2 M} \sum c_{i j}^{2} g_{j}+\sum a_{i j}^{2} g_{j}+\left(b+\eta^{2}|x|^{2 M} d\right) f$.

REMARK. If there are many $g_{j}$ in Theorem 10.1, the set of $g_{(J)}$ can be quite large. In this case, we can give an alternative characterization of the set $S$ provided the hypotheses of Theorem 10.11 hold. If so, we can find $\varepsilon>0$ such that $\varphi(x)>\varepsilon^{2}$ for all $x \in X$ because $X$ is compact. Choose a representation of $\varphi$ as in Theorem 10.11 and apply Theorem 10.1 to $h$. Therefore, under the hypotheses of Theorem 10.11 we will have $\varphi(x)>0$ for all $x \in X$ if and only if $\varphi$ satisfies an equation of the form

$$
k\left[\varphi-\varepsilon^{2}-\sum a_{i j}^{2} g_{j}-b f\right]=1+\sum k_{v}^{2}
$$

all terms except $\varepsilon$ being polynomials. 
If $X$ is a finite simplicial complex or, more generally, a finite union $X=\cup X_{i}$ where the $X_{i}$ are as in Theorem 10.1, the results obtained here do not apply directly to $X$ but we can apply them to each $X_{i}$ to arrive at an algebraic description of the polynomial functions which are never zero on $X$.

It is perhaps worth remarking that the hypothesis of Theorem 10.11 applies to the usual presentation of a simplex: $x_{i} \geqslant 0, \Sigma x_{i} \leqslant 1$. In fact,

$$
\sum_{i}\left[\sum_{j \neq i} x_{j}^{2}+\left(x_{i}-1\right)^{2}\right] x_{i}+\left(1+\sum x_{j}^{2}\right)\left(1-\sum x_{i}\right)=1-\sum x_{i}^{2} .
$$

Problem. An interesting question is whether Hilbert's 17th problem has an affirmative solution in the ring $A=R_{S}$. In particular, if $f$ is a polynomial with $f(x) \geqslant 0$ for all $x \in \mathbf{R}^{n}$, can one find polynomials $h$ and $h_{i}$ such that $h(x) \neq 0$ for all $x \in \mathbf{R}^{n}$ and $h^{2} f=\sum h_{i}^{2}$ ?

Write $\Sigma=\left\{1+\sum a_{i}^{2}\right\}$ and $\Pi=\left\{\Sigma b_{j}^{2}\right\}$ where the $a_{i}$ and $b_{j}$ are polynomials. If $h$ is never zero we can write $h k=s \in \Sigma$ by Theorem 10.1. Therefore, an equivalent question is whether one can write $s f=p$ with $s \in \Sigma$ and $p \in \Pi$.

If we assume $f(x)>0$ for all $x \in \mathbf{R}^{n}$, there are a number of equivalent formulations of this problem. Namely one can look for representations of $f$ in one of the following forms (a) $s f=p$, (b) $s f=a^{2} s^{\prime}$, (c) $g^{2} f=s$, (d) $p f=s$ where $s, s^{\prime} \in \Sigma, p \in \Pi, a \in \mathbf{R}, a \neq 0$. These are equivalent in the sense that if every $f>0$ on $\mathbf{R}^{n}$ admits a representation in one of these forms then every such $f$ admits a representation in each of these forms.

To see this, replace $f$ by $a^{2}\left(1+|x|^{2}\right)^{n} f$. By Corollary 10.8 we can then assume $f \geqslant 2$. If (a) holds, write $s(f-1)=p$ and get (b) $s f=s+p=s^{\prime}$ $\in \Sigma$. If (b) holds, then (c) $s^{2} f=s s^{\prime} \in \Sigma$. Clearly (c) implies (d). If (d) holds, then (a) $s f=p f^{2} \in \Pi$.

A related problem is whether Hilbert's 17 th problem has an affirmative solution in the local ring of a point i.e., if a polynomial satisfies $f \geqslant 0$ (or even $f>0$ ) on all of $\mathbf{R}^{n}$ and $a \in \mathbf{R}^{n}$ is given can one write $h^{2} f=\sum h_{i}^{2}$ with $h(a) \neq 0, h$ and $h_{i}$ being polynomials.

ADDED IN PROOF. In their paper An old question of Hilbert, Proc. of Quad. Form Conf., Queen's Univ., 1976 (to appear), M. D. Choi and T. Y. Lam have announced that the solution to these problems is negative in general but is positive in the case $f>0$. Their proof for $f>0$ applies to the situation of Theorem 10.1: apply Theorem 10.1 to $u^{2}+f$ on $X \times R$ and use the argument of Habicht, Comm. Math. Helv. 12 (1940), 317-322. A. Prestel also pointed out that the case $f>0$ follows from Theorem 5.10 of his Lectures on formally real fields, IMPA, Rio de Janeiro, 1975.

11. The sphere. By combining the theory developed above with a result of Fossum [15] we can obtain a purely algebraic description of the homotopy 
groups of the classical Lie groups. Unfortunately, this result seems to be completely useless for computation of these groups.

For a commutative ring $A$ let $P_{r}(A)$ be the set of isomorphism classes of finitely generated projective $A$-modules of rank $r$. If $A$ is an R-algebra and $P$ is a finitely generated projective $\mathbf{H} \otimes_{\mathrm{R}} A$-module, we can define the rank of $P$ to be $1 / 4$ of the rank of $P$ as an $A$-module. If $A$ has an ideal $M$ with $A / M=\mathbf{R}$, then $P / M P$ is an $\mathrm{H}$-module so that $4 \mid \mathrm{rk}_{n} P$. Therefore if $P$ has constant rank over $A$, this rank will be divisible by 4 and the rank as defined above will be an integer. The set $P_{r}\left(\mathbf{H} \otimes_{\mathbf{R}} A\right)$ is defined as above using this rank.

THEOREM 11.1. Let $R=\mathbf{R}\left[x_{0}, \ldots, x_{n}\right] /\left(\sum x_{i}^{2}-1\right)$, let $S=\left\{1+\sum r_{i}^{2} \mid r_{i}\right.$ $\in R\}$ and let $A=R_{S}$. Then there are bijections

(a) $P_{r}(A) \rightarrow \pi_{n}(B O(r))$,

(b) $P_{r}\left(\mathbf{C} \otimes_{\mathrm{R}} A\right) \rightarrow \pi_{n}(B U(r))$,

(c) $P_{r}\left(\mathbf{H} \otimes_{\mathrm{R}} A\right) \rightarrow \pi_{n}(B S p(r))$.

The maps here are induced by the inclusion $A \hookrightarrow \mathbf{R}^{S^{n}}$, the groups on the right being isomorphic to $P_{r}\left(\mathbf{R}^{S^{n}}\right), P_{r}\left(\mathbf{C}^{S^{n}}\right)$, and $P_{r}\left(\mathbf{H}^{S^{n}}\right)$ by [42] and the classification of bundles over $S^{n}$ [41], [25]. Parts (a) and (b) follow immediately from Fossum's result [15] and Theorem 2.2. The case $n=2$ (with $S$ $=\left\{f \in R \mid f \neq 0\right.$ on $\left.\left.S^{2}\right\}\right)$ was done by Moore [31] using very explicit methods. Similar results for projective spaces (but without the interpretation in terms of homotopy groups) can be deduced from the results of [16]. Presumably these results also extend to the quaternionic case but I have not checked this.

To prove (c), it is necessary to extend Fossum's result to the quaternionic case. In order to do this, it will first be necessary to extend the results of [3] to this case. I will assume the reader is familiar with the methods, results, and notation of [3] (see also [25, Chapter 11]).

Let $C_{n}$ be the Clifford algebra over $\mathbf{R}$ defined by the quadratic form $-|x|^{2}=-\sum_{1}^{n} x_{i}^{2}$. Let $\Lambda=\mathbf{R}, \mathbf{C}$, or $\mathbf{H}$ and consider $\Lambda \otimes_{\mathrm{R}} C_{n}$ graded by $\Lambda \otimes_{\mathrm{R}} C_{n}^{0}+\Lambda \otimes_{\mathrm{R}} C_{n}^{1}$. A finitely generated graded $\Lambda \otimes C_{n}$-module $M$ consists of two finite-dimensional $\Lambda$-modules $M^{0}$ and $M^{1}$ together with linear maps $\varphi: \mathbf{R}^{n} \rightarrow \operatorname{Hom}_{\Lambda}\left(M^{1}, M^{0}\right)$ and $\psi: \mathbf{R}^{n} \rightarrow \operatorname{Hom}_{\Lambda}\left(M^{0}, M^{1}\right)$ such that $\varphi(x) \psi(x)$ $=-|x|^{2}=\psi(x) \varphi(x)$. If we regard $\mathbf{R}^{n}$ as a subspace of $C_{n}$ then $\varphi(x), \psi(x)$ are simply multiplication by $x$.

Let $e_{1}, \ldots, e_{n}$ be the canonical base for $\mathbf{R}^{n}$ and write $\varphi_{i}=\varphi\left(e_{i}\right), \psi_{i}=\psi\left(e_{i}\right)$ so that $\varphi(x)=\sum x_{i} \varphi_{i}, \psi(x)=\sum x_{i} \psi_{i}$. Let $R$ be as in Theorem 11.1 and define an endomorphism $\alpha$ of $R \otimes_{\mathbf{R}} M$ by setting, as in [15],

$$
\alpha\left(m^{0}, m^{1}\right)=\frac{1}{2}\left[\left(1-x_{0}\right) m^{0}+\psi(x) m^{1},-\psi(x) m^{0}+\left(1+x_{0}\right) m^{1}\right]
$$

where $\left(m^{0}, m^{1}\right) \in R \otimes_{\mathbf{R}} M^{0} \oplus R \otimes_{\mathbf{R}} M^{1}$ and $\varphi(x), \psi(x)$ mean $\sum_{1}^{n} x_{i} \varphi_{i}$ and 
$\sum_{1}^{n} x_{i} \psi_{i}$. In matrix form,

$$
\alpha=\frac{1}{2}\left(\begin{array}{cc}
1-x_{0} & \varphi(x) \\
-\psi(x) & 1+x_{0}
\end{array}\right) .
$$

It is easy to check that $\alpha$ is idempotent. Define $c(M)=\operatorname{ker} \alpha$. This is a finitely generated projective $\Lambda \otimes R$-module of rank equal to that of $M^{0}$. Let $M_{n}(\Lambda)$ be $K_{0}$ of the category of finite-dimensional graded $\Lambda \otimes C_{n}$-modules. By sending $M$ to $[c(M)]-\left[R \otimes M^{0}\right]$ we get a homomorphism $M_{n}(\Lambda) \rightarrow \tilde{K}_{0}(\Lambda \otimes R)$. Composing this with the map induced by $\Lambda \otimes R \rightarrow \Lambda^{S^{n}}$ gives $\alpha_{\Lambda}^{\prime}: M_{n}(\Lambda)$ $\rightarrow \tilde{K}_{0}\left(\Lambda^{S^{n}}\right)=\tilde{K}_{\Lambda}^{0}\left(S^{n}\right)$. To show that $\tilde{K}_{0}(\Lambda \otimes R) \rightarrow \tilde{K}_{\Lambda}^{0}\left(S^{n}\right)$ is onto, it will suffice to do this for $\alpha_{\Lambda}^{\prime}$. As Fossum observes [15], $\alpha_{\Lambda}^{\prime}$ agrees with the map defined in $[3, \S 11]$. To see this, note that if $\alpha\left(m^{0}, m^{1}\right)=0$ then, on $U^{-}$ $=\left\{x \in S^{n} \mid x_{0} \neq 1\right\}$ we have $m^{0}=-\varphi(x) m^{1} /\left(1-x_{0}\right)$ and on $U^{+}=\{x$ $\left.\in S^{n} \mid x_{0} \neq-1\right\}$ we have $m^{1}=\psi(x) m^{0} /\left(1+x_{0}\right)$. Therefore, the corresponding bundle is identified with $U^{-} \times M^{1}$ over $U^{-}$and $U^{+} \times M^{0}$ over $U^{+}$. By comparing these representations on the equator $x_{0}=0$ we see that the bundle is defined by the clutching function $\varphi(x)$ as in $[3, \S 11]$.

If $M$ is a $\Lambda \otimes C_{n+1}$-module and $M^{\prime}$ is $M$ considered as an $\Lambda \otimes C_{n}$-module, it is clear that the bundle on $S^{n+1}$ defined by $c(M)$ restricts to the bundle on $S^{n}=\left\{x_{n+1}=0\right\}$ defined by $c\left(M^{\prime}\right)$. Consequently, this bundle is trivial. Therefore, if $A_{n}(\Lambda)$ is the cokernel of the map $M_{n+1}(\Lambda) \rightarrow M_{n}(\Lambda)$ sending $M$ to $M^{\prime}$, then $\alpha_{\Lambda}^{\prime}$ induces $\alpha_{\Lambda}: A_{n}(\Lambda) \rightarrow \tilde{K}_{\Lambda}^{0}\left(S^{n}\right)$.

For $\Lambda=\mathbf{R}$ or $\mathbf{C}$, we can define products $M_{m}(\Lambda) \otimes M_{n}(\Lambda) \rightarrow M_{m+n}(\Lambda)$ by sending $[M] \otimes[N]$ to $[M \hat{\otimes} N]$ using $C_{m} \hat{\otimes} C_{n}=C_{m+n}$, the hat denoting graded tensor product as in [3]. For $\mathbf{H}$ we get $M_{m}(\mathbf{R}) \otimes M_{n}(\mathbf{H}) \rightarrow M_{m+n}(\mathbf{H})$ by $[M] \otimes[N] \mapsto\left[M \otimes_{\mathbf{R}} N\right]$ and $M_{m}(\mathbf{H}) \otimes M_{n}(\mathbf{H}) \rightarrow M_{m+n}(\mathbf{R})$ by $[M] \otimes[N]$ $\mapsto\left[M \otimes_{\mathrm{H}} N\right]$. These induce similar products on the $A_{n}(\Lambda)$. The same construction works for vector bundles giving products $\tilde{K}_{\Lambda}^{0}\left(S^{m}\right) \otimes \tilde{K}_{\Lambda}^{0}\left(S^{n}\right)$ $\rightarrow \tilde{K}_{\Lambda}^{0}\left(S^{m} \wedge S^{n}\right)$ for $\Lambda=\mathbf{R}$ or $\mathbf{C}$ as in $[3, \S 11]$ and $\tilde{K}_{\mathbf{R}}^{0}\left(S^{m}\right) \otimes \tilde{K}_{\mathbf{H}}^{0}\left(S^{n}\right)$ $\rightarrow \tilde{K}_{\mathbf{H}}^{0}\left(S^{m} \wedge S^{n}\right)$ and $\tilde{K}_{\mathbf{H}}^{0}\left(S^{m}\right) \otimes \tilde{K}_{\mathbf{H}}^{0}\left(S^{n}\right) \rightarrow \tilde{K}_{\mathbf{R}}^{0}\left(S^{m} \wedge S^{n}\right)$. The proof of $[3$, Proposition 11.1] shows that $\alpha_{\Lambda}$ preserves all these products.

In [3, Theorem 11.5] it is shown that $\alpha_{\Lambda}$ is an isomorphism for $\Lambda=\mathbf{R}$ or $\mathbf{C}$.

TheOREM 11.2. $\alpha_{\mathbf{H}}: A_{n}(\mathbf{H}) \approx \tilde{K}_{\mathbf{H}}^{0}\left(S^{n}\right)$.

Proof. The first step is to show that the groups in question are isomorphic. For $\tilde{K}_{\mathbf{H}}^{0}\left(S^{n}\right)=\pi_{n}(B S p)$, use Bott's calculations [8]. To compute $A_{n}(\mathbf{H})$, first observe that by [3, Proposition 5.1] every $\mathbf{H} \otimes C_{n}$-module $M$ has the form $C_{n} \otimes_{C_{n}^{0}} M^{0}$ where $M^{0}$ is an $\mathbf{H} \otimes C_{n}^{0}$-module. By [3, Proposition 5.4] $\mathbf{H} \otimes C_{n}^{0}$ $\approx \mathbf{H} \otimes C_{n-1}$. Using the values of $C_{k}$ given in $[3, \S 5]$ it is easy to determine the number $N_{n}$ of simple graded $\mathbf{H} \otimes C_{n}$-modules and their dimensions $2 a_{n}$ (note that $\operatorname{dim} M^{0}=\operatorname{dim} M^{\prime}$ ). If $N_{n}==1$ and $a_{n}=a_{n+1}$ then $A_{n}(\mathbf{H})=0$ 
since $M_{n}(\mathbf{H})=\mathbf{Z}$ and, if $N$ is a simple $\mathbf{H} \otimes C_{n+1}$-module, its restriction $N^{\prime}$ to $\mathbf{H} \otimes C_{n}$ has dimension equal to that of a simple $\mathbf{H} \otimes C_{n}$-module $M$ and so $M \approx N^{\prime}$. If $N_{n}=1$ and $a_{n+1}=2 a_{n}$ then $N^{\prime}$ must contain 2 copies of $M$ and $A_{n}(\mathrm{H})=\mathrm{Z} / 2 \mathrm{Z}$. In case $N_{n}=2$, it turns out that $N_{n+1}=1$ and $a_{n+1}=2 a_{n}$. If $M_{1}$ and $M_{2}$ are the simple $\mathbf{H} \otimes C_{n}$-modules and $N$ is the simple $\mathbf{H} \otimes C_{n+1^{-}}$ module, then $N^{\prime}$ must be $2 M_{1}, 2 M_{2}$ or $M_{1} \oplus M_{2}$. But $C_{n+1}$ is a free $C_{n}$-module. Therefore both $M_{1}$ and $M_{2}$ occur in $\mathbf{H} \otimes C_{n+1}$ considered as an $\mathbf{H} \otimes C_{n^{-}}$ module. But $\mathbf{H} \otimes C_{n+1}$ is a sum of copies of $N$ so $M_{1}$ and $M_{2}$ occur in $N^{\prime}$ and $N^{\prime} \approx M_{1} \oplus M_{2}$. Therefore, $A_{n}(\mathrm{H})=\mathrm{Z}$ in this case.

Now let $M^{0}=M^{1}=\mathbf{H}$. After identifying $\mathbf{R}^{4}$ with $\mathbf{H}$ we define $\varphi$ and $\psi$ by $\varphi(x) y=y x, \psi(x) y=-y x^{*}$ where $x \mapsto x^{*}$ is the usual involution on $\mathbf{H}$. This makes $M=M^{0} \oplus M^{1}$ a graded $\mathbf{H} \otimes C_{4}$-module. By checking dimensions it is seen to be simple. Let $\mu \in A_{4}(\mathbf{H})$ be the class of this module. By checking dimensions again we see that $M \otimes_{\mathrm{H}} M$ is a simple $C_{8}$-module and so represents the element $\lambda \in A_{8}(\mathbf{R})$ of [3, Corollary 6.6]. In other words $\mu \mu=\lambda$ under the product $A_{4}(\mathrm{H}) \otimes A_{4}(\mathrm{H}) \rightarrow A_{8}(\mathrm{R})$. By taking products with $\mu$ we get $A_{n}(\mathbf{R}) \rightarrow^{\mu} A_{n+4}(\mathrm{H}) \rightarrow A_{n+8}(\mathbf{R})$. The composition is multiplication by $\lambda$ which is an isomorphism by [3, Proposition 6.8]. Since the groups involved are all isomorphic and are $0, \mathbf{Z} / 2 \mathbf{Z}$, or $\mathbf{Z}$, it follows that $A_{n}(\mathbf{R}) \rightarrow^{\mu} A_{n+4}(\mathbf{H})$ and $A_{n}(\mathbf{H}) \rightarrow^{\mu} A_{n+4}(\mathbf{R})$ are isomorphisms (the second case being trivial for $n<4)$.

Now consider the diagram

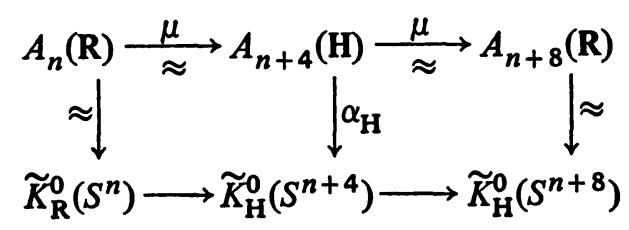

where the maps on the bottom are multiplication by the image of $\mu$. Since all groups in this diagram are isomorphic and are $0, \mathbf{Z} / 2 \mathbf{Z}$, or $\mathbf{Z}$, it follows that all maps in the diagram are isomorphisms.

REMARK. The calculations of [42] show that the image of $\mu$ in $\tilde{K}_{\mathbf{H}}^{0}\left(S^{4}\right)$ is represented by the canonical line bundle over $\mathbf{P}^{1}(\mathbf{H})=S^{4}$. This is, of course, the bundle associated with the Hopf bundle $S^{7} \rightarrow S^{4}$

The group structure on $P_{r}(A)$, etc. induced by the bijections of Theorem 11.1 can also be calculated algebraically. Let $U=\left\{1+\sum r_{i}^{2}+x_{0} \sum s_{j}^{2} \mid r_{i}, s_{j} \in R\right\}$ and $V=\left\{1+\sum r_{i}^{2}-x_{0} \sum s_{j}^{2} \mid r_{i}, s_{j} \in R\right\}$. Then $R_{U}$ and $R_{V}$ are the rings associated to the upper and lower hemispheres of $S^{n}$ by Theorem 10.1. By Theorem 2.2, all projective modules over $R_{U}$ and $R_{V}$ are free. Let $P$ and $Q$ be finitely generated projective $A$-modules of rank $r$. Let $F=A^{r}$. Then $P_{U} \approx F_{U}$ and $Q_{V} \approx F_{V}$ so we can find $\alpha: P \rightarrow F, \beta: Q \rightarrow F$ such that $\alpha_{U}, \beta_{V}$ are 
isomorphisms [33, Lemma 2.2b]. Let $M$ be the cokernel of $P \oplus Q \rightarrow^{(\alpha, \beta)} F$. We can find $u \in U$ and $v \in V$ such that $u M=0$ and $v M=0$. Therefore $\left(u^{2}+v^{2}\right) M=0$. But $u^{2}+v^{2}$ is never zero on $S^{n}$ and hence is a unit of $A$ so $M=0$. Let $T$ be the kernel of $(\alpha, \beta): P \oplus Q \rightarrow F$. Then $T$ is a finitely generated projective $A$-module of rank $r$ and the group operation on $P_{r}(A)$ is given by $[P][Q]=[T]$. The same construction works for $\mathbf{C} \otimes A$ and $\mathbf{H} \otimes A$.

To prove this result, it suffices to check the analogous statement for vector bundles. It is easy to check, by choosing explicit trivializations over the two hemispheres, that the construction corresponds to taking products of clutching functions.

Note that it will not do to take any epimorphism $P \oplus Q \rightarrow F$. For example, if $P$ is the tangent bundle then $P \oplus F \approx F \oplus F$ so we can find an epimorphism $F \oplus F \rightarrow F$ with kernel $P \approx F$.

It might be of some interest to find purely algebraic conditions under which a construction similar to that given here makes $P_{r}(A)$ into an abelian group.

\section{REFERENCES}

1. E. Artin, Uber die Zerlegung definiter Funktionen in Quadrate, Abh. Math. Sem. Univ. Hamburg 5 (1927), 100-115.

2. M. F. Atiyah and R. Bott, On the periodicity theorem for complex vector bundles, Acta Math. 112 (1964), 229-247. MR 31 \#2727.

3. M. F. Atiyah, R. Bott and A. Shapiro, Clifford modules, Topology 3 (1964), 3-38. MR 29 \# 5250 .

4. M. F. Atiyah and F. Hirzebruch, Vector bundles and homogeneous spaces, Differential Geometry (Proc. Sympos. Pure Math., vol. 3), Amer. Math. Soc., Providence, R.I., 1961, pp. 7-38. MR 25 \#2617.

5. H. Bass, An algebraic analogue of Bott's complex periodicity theorem, Mimeographed notes, Columbia Univ., 1964.

6. - Algebraic K-theory, Benjamin, New York, 1968. MR 40 \#2736.

7. - Modules which support nonsingular forms, J. Algebra 13 (1969), 246-252. MR 39 \#6875.

8. R. Bott, The stable homotopy of the classical groups, Ann. of Math. (2) 70 (1959), 313-337. MR 22 \#987.

9. B. H. Dayton, K-theory of special normed algebras (to appear).

10. D. W. Dubois and G. Efroymson, Algebraic theory of real varieties. I, Studies and essays (Presented to Yu-why Chen on his 60th birthday), Math. Res. Center, Nat. Taiwan Univ., Taipei, 1970, pp. 107-135. MR 43 \#6203.

11. E. Dyer, Cohomology theories, Benjamin, New York, 1969. MR 42 \#3780.

12. S. Eilenberg and N. E. Steenrod, Foundations of algebraic topology, Princeton Univ. Press, Princeton, N.J., 1952. MR 14 \# 398.

13. E. G. Evans, Jr., Projective modules as fiber bundles, Proc. Amer. Math. Soc. 27 (1971), 623-626. MR 42 \#4537.

14. O. Forster, Funktionentheoretischer Hilfsmittel in der Theorie der kommutativen Banach Algebren, Jber. Deutsch. Math.-Verein 76 (1974), 1-17.

15. R. Fossum, Vector bundles over spheres are algebraic, Invent. Math. 8 (1969), 222-225. MR 40 \#3537.

16. A. V. Geramita and L. G. Roberts, Algebraic vector bundles on projective space, Invent. Math. 10 (1970), 298-304. 
17. L. Gillman and M. Jerison, Rings of continuous functions, Van Nostrand, Princeton, N.J., 1960. MR 22 \#6994.

18. E. A. Gorin, Asymptotic properties of polynomials and algebraic functions of several variables, Uspehi Mat. Nauk 16 (1961), 91-118 = Russian Math. Surveys 6 (1961), 93-119. MR 24 \# A1269.

19. D. Gondard and P. Ribenboim, Fonctions définies positives sur les variétés réelles, Bull. Sci. Math. 98 (1974), 39-47.

20. A. Grothendieck, Revêtements étales et groupe fondamental, SGA1, Lecture Notes in Math., vol. 224, Springer-Verlag, Berlin and New York, 1971. MR 50 \#129.

21. D. Hilbert, Über die Darstellung definiter Formen als Summe von Formenquadraten, Math. Ann. 32 (1888), 342-350.

22. L. Hörmander, On the division of distributions by polynomials, Ark. Mat. 3 (1958), 555-568. MR 23 \# A2044.

23. S. T. Hu, Homotopy theory, Academic Press, New York, 1959. MR 21 \# 5186.

24. W. Hurewicz and H. Wallman, Dimension theory, Princeton Univ. Press, Princeton, N.J., 1941. MR 3, 312.

25. D. Husemoller, Fibre bundles, McGraw-Hill, New York, 1966. MR 37 \#4821.

26. N. Jacobson, Structure of rings, Amer. Math. Soc. Colloq. Publ.,Vol. 37, Providence, R.I., 1964. MR 36 \#5158.

27. I. Kaplansky, Commutative rings, Allyn and Bacon, Boston, Mass., 1970. MR 40 \# 7234.

28. K. Lønsted, An algebraization of vector bundles on compact manifolds, J. Pure Appl. Algebra 2 (1972), 193-207. MR 49 \#2733.

29. - Vector bundles over finite $\mathrm{CW}$ complexes are algebraic, Proc. Amer. Math. Soc. 38 (1973), 27-31. MR 47 \# 5862.

30. L. H. Loomis, An introduction to abstract harmonic analysis, Van Nostrand, New York, 1953. MR 14, 883.

31. N. Moore, Algebraic vector bundles over the 2-sphere, Invent. Math. 14 (1971), 167-172. MR 45 \#3403.

32. T. S. Motzkin, The arithmetic-geometric inequality, Inequalities (Proc. Sympos. WrightPatterson Air Force Base, Ohio, 1965), Academic Press, New York, 1967, pp. 205-224. MR 36 \#6569.

33. M. P. Murthy and R. G. Swan, Vector bundles over affine surfaces, Invent. Math. 36 (1976), $125-165$.

34. K. Nogami, Dimension theory, Academic Press, New York, 1970.

35. M. Nagata, Local rings, Interscience, New York, 1962. MR 27 \#5790.

36. A. Robinson, Complete theories, North-Holland, Amsterdam, 1956. MR 17, 817.

37. Introduction to model theory and to the metamathematics of algebra, North-Holland, Amstcrdam, 1963. MR 27 \#3533.

38. R. M. Robinson, Some definite polynomials which are not sums of squares of real polynomials, Selected Questions of Algebra and Logic (Volume dedicated to the memory of A. J. Mal'cev), Izdat. "Nauka" Sibirsk. Otdel., Novosibirsk, 1973, pp. 264-282. MR 49 \#2647.

39. A. Seidenberg, On the dimension theory of rings. II, Pacific J. Math. 4 (1954), 603-614. MR 16, 441.

40. J.-P. Serre, Groupes d'homotopie et classes de groupes abéliens, Ann. of Math. (2) 58 (1953), 258-294. MR 15, 548.

41. N. E. Steenrod, The topology of fiber bundles, Princeton Univ. Press, Princeton, N.J., 1951. MR 12, 522.

42. R. G. Swan, Vector bundles and projective modules, Trans. Amer. Math. Soc. 105 (1962), 264-277. MR 26 \#785.

43. - Algebraic $K$-theory, Lecture Notes in Math., vol. 76, Springer-Verlag, Berlin and New York, 1968. MR 39 \#6940.

44. - A cancellation theorem for projective modules in the metastable range, Invent. Math. 27 (1974), 23-43. 
45. - Serre's problem, Report of the Conference in Algebra, Queen's Papers in Pure and Appl. Math. No. 42, Queen's Univ., Kingston, Ont., 1975.

46. L. N. Vaserštenn, Stable range of rings and dimensionality of topological spaces, Funkcional. Anal. i Prilozen. 5 (1971), no. 2, 17-27 = Functional Anal. Appl. 5 (1971), 102-110. MR 44 \#1701.

47. H. Whitney, Elementary structure of real algebraic varieties, Ann. of Math. (2) 66 (1957), 545-556. MR 20 \#2342.

48. O. Zariski and P. Samuel, Commutative algebra, vol. I, Van Nostrand, Princeton, N.J., 1958. MR 19, 833.

Department of Mathematics, University of Chicago, Chicago, Illinois 60637 